University of Warwick institutional repository: http://go.warwick.ac.uk/wrap This paper is made available online in accordance with publisher policies. Please scroll down to view the document itself. Please refer to the repository record for this item and our policy information available from the repository home page for further information.

To see the final version of this paper please visit the publisher's website. Access to the published version may require a subscription.

Author(s): D. B. A. EPSTEIN, A. MARDEN and V. MARKOVIC Article Title: CONVEX REGIONS IN THE PLANE AND THEIR DOMES Year of publication: 2006 Link to published version: http://dx.doi.org/ 10.1017/S002461150501573X

Publisher statement: None 
Proc. London Math. Soc. (3) 92 (2006) 624-654 $\quad$ (C) 2006 London Mathematical Society doi:10.1112/S002461150501573X

\title{
CONVEX REGIONS IN THE PLANE AND THEIR DOMES
}

\author{
D. B. A. EPSTEIN, A. MARDEN AND V. MARKOVIC
}

\section{Introduction}

Let $\Omega$ be a euclidean convex region not equal to $\mathbb{C}$. It need not have compact closure. We will study $\Omega$ from above by examining its geometric relationship to $\operatorname{Dome}(\Omega)$. Here Dome $(\Omega)$ is the relative boundary component in the upper half 3 -space of the hyperbolic convex hull of the closed set $\mathbb{S}^{2} \backslash \Omega$. It is constructed by considering maximal disks whose interiors are contained in $\Omega$. Each is bounded by a circle $c$ meeting the boundary at two or more points. From the circle $c$ rises a hemisphere $h$ which divides upper half-space into two components. Denote the component which does not abut the disk by $H_{h}$. The hyperbolic convex hull is $\bigcap_{h} H_{h}$. The dome is the envelope of the hemispheres. In short, the dome lies over the floor $\Omega$ as the dome of a domed stadium lies over the ground under it.

The ambient hyperbolic metric induces a path metric on the dome, which is referred to as the hyperbolic metric on the dome. Thurston proved the Riemann mapping theorem for it, namely there is an isometry between the dome and the unit disk in the respective hyperbolic metrics. It is uniquely determined up to compositions by isometries.

In more detail, the dome is the union of at most countably many open flat pieces, which are hyperbolic polygons generally with infinitely many sides, and the complementary closed set. The complementary set is the union of mutually disjoint bending lines. Each bending line is a hyperbolic geodesic with endpoints in the common boundary $\partial \Omega$ of $\Omega$ and $\operatorname{Dome}(\Omega)$.

An isolated bending line $\ell$ has a well-defined bending angle $\theta$ which we take to be the exterior bending angle. Thus $0<\theta<\pi$ where the extreme $\theta=0$ means no bending at all, while $\theta=\pi$ means one side of $\ell$ is folded over the other. In general, there is a real-valued Borel measure $\mu$ on transverse segments $\tau$ whose endpoints are in flat pieces or on bending lines with zero atomic measures. It is constructed in a manner akin to the Riemann integral by using finite approximations to the dome. Its value depends only on the flat pieces or lines containing the end points of $\tau$.

For example, if $\Omega$ is a wedge with interior vertex angle less than $\pi$, its dome is a half-cone so there are no flat pieces. If $\Omega$ is a triangle, its dome is the union of three conical pieces and a flat piece. In fact the dome is a $C^{1}$-surface without any isolated bending lines. Similar statements are true for any convex polygon (finite sided).

For an introduction to the general theory see $[\mathbf{1}]$.

The basic tool we use is the nearest point retraction $r: \Omega \rightarrow \operatorname{Dome}(\Omega)$. This is defined as follows. Given $z \in \Omega$ consider the family of spheres in upper half-space which are tangent to $\mathbb{C}$ at $z$. As the diameter increases there is a first diameter

Received 18 August 2004; revised 11 July 2005.

2000 Mathematics Subject Classification 30C75, 30F40, 30F45, 30F60. 
where the sphere touches the dome, necessarily at a single point which we denote by $r(z)$. The retraction $r$ is continuous, and is a homeomorphism if and only if there are no isolated bending lines. In any case $r$ extends continuously to $\partial \Omega$ and pointwise fixes it.

There are two theorems proved in this paper for euclidean convex regions. The first is Theorem 5.1. It states that $r$ is actually a 2-quasiconformal homeomorphism.

The second, Theorem 7.1, gives a universal bound on the distortion of $r$ at the boundary. At least for convex regions with $C^{1}$-boundaries, this result implies that there exists a unique extremal quasiconformal map $f$ from the floor to the dome $\Omega$ which, like $r$, pointwise fixes the common boundary. Moreover, $f$ is of Teichmüller type, that is, $f_{\bar{z}} / f_{z}=k \phi /|\phi|$ for some quadratic differential $\phi d z^{2}$ with $\iint|\phi|<\infty$ and constant $0 \leqslant k<1$. We also show that the maximal dilatation of the extremal mapping cannot exceed 2 .

In spite of the transparency of our results for finite-sided convex polygons, there are major technical hurdles in extending the results, especially for the second theorem whose proof occupies most of this paper. We were not at all sure what was true when we started our investigation.

Our results about convex regions of the plane can be made invariant under Möbius transformations, by suitably generalizing the class of regions.

Let $\Omega \subset \mathbb{S}^{2}$ be a simply connected open region, not the complement of a point. For each boundary point $p$, we suppose that $p$ has a neighbourhood, such that $\partial \Omega$ near $p$ is locally the graph of a function $f_{p}$. We assume that the second derivative of $f_{p}$ in the distributional sense is a constant plus a positive Borel measure. In this case we say that $\Omega$ is infinitesimally convex. We thank Adrien Douady for making this condition explicit for us. The condition is invariant under Möbius transformations.

If $\Omega$ is an infinitesimally convex set, given $p \in \partial \Omega$, there exists a Möbius transformation that sends a neighbourhood of $p$ onto a convex set. Our proof will then apply. On the other hand, a region invariant under a non-elementary discrete group cannot be infinitessimally convex unless it is a round disk.

We are very grateful to the referee for a careful reading of the manuscript.

\section{Outline of the paper}

As stated above, $\Omega$ will be a proper convex open subset of $\mathbb{C}$ (with a few explicit exceptions). We allow $\Omega$ to have non-compact closure. In Theorem 5.1, we show that $r: \Omega \rightarrow$ Dome $(\Omega)$, the nearest point retraction, is a quasiconformal homeomorphism with quasiconformal constant at most 2. This proves Thurston's $K=2$ conjecture for euclidean convex regions. The conjecture is false for certain non-convex regions; see $[\mathbf{2}, \mathbf{3}]$. In Definition 6.6, we recall the definition of substantial boundary dilatation. In Theorem 7.1, we compute the substantial boundary dilatation of $r$ at each point of $\partial \Omega$. This enables us to use the method of Chris Bishop to show that the Makarov Integral Means Spectrum for a euclidean convex region covers a larger range of exponents than that predicted by the Brennan Conjecture. In fact, the application to Brennan's conjecture does not need the substantial boundary dilatation, because the derivative of the Riemann mapping of a convex domain to the unit disk is uniformly bounded. (We thank C. Pommerenke for pointing this out to us.)

Our main objective is to study the substantial boundary dilatation at a point $p \in \partial \Omega$. So we are only interested in the situation near $p$, and it is reasonable to 
'zoom in'. This method is discussed more formally in $\S 4$. We zoom in as follows. We take a small round circle $C$, centred at $p$. This makes sense also if $p=\infty \in \mathbb{S}^{2}$. Let $A=\Omega \cap C$. We apply a similarity to $\mathbb{C}$, so that $A$ becomes an arc of unit length, with the midpoint of $A$ at the origin, and tangent direction at the midpoint vertical. Then, as $C$ converges to $p, \Omega$ converges either to a wedge or to an infinite strip of width 1 .

We therefore study first, as important special cases of convex regions, wedges and strips, together with their domes. This study starts with Lemma 3.1 and finishes with Corollary 4.4. We also discuss some standard homeomorphisms between them. These standard isomorphisms provide a model that we try to follow in more complicated situations. In particular, there are canonical conformal isomorphisms of wedges, of half-cone surfaces and of half-cylinder surfaces, with the infinite strip of width 1 .

Theorem 4.6 shows that the dome of a convex open region is a $C^{1}$-submanifold of $\mathbb{H}^{3}$. A convex polygon with a finite number of sides breaks up canonically into a finite number of real analytic pieces, each of which corresponds, under the nearest point retraction, to some conical or hemispherical piece in the dome (see the proof of Theorem 5.1). From this we deduce (Theorem 5.1) that the dilatation of the nearest point retraction for an arbitrary convex region has dilatation bounded above by 2 .

The Approximation Theorem (Theorem 5.2), proved in [3], is an essential tool in arguing that the finite-sided case implies the general case. We prove the well-known fact that there is a well-defined angle at each point of the boundary of a general convex region in the plane (from Definition 6.1 to Remark 6.5).

Next, we state the main result of this section (Theorem 7.1), together with the one necessary additional definition (Definition 6.6). In $\S 7.2$, Theorem 7.1 is proved in some special situations. In $\S 7.3$, we specify how to move $\Omega$ by a similarity, so that its position becomes standard. This is useful when one works in coordinates.

In $\S 7.6$, we present a rather explicit approximation of $\Omega$ by a region $\Omega_{n}$, which, if $p=\infty$, is a locally finite convex polygon. If $p$ is finite, $\partial \Omega_{n}$ is locally finite except in a neighbourhood of $p$. It turns out that the proof of Theorem 7.1 is easier when $\Omega$ is replaced by $\Omega_{n}$. After proving the result for $\Omega_{n}$, we can deduce it for $\Omega$ by letting $n$ tend to infinity. In order to make $\Omega$ and $\Omega_{n}$ more similar to a strip, we apply log, getting subspaces $M$ and $M_{n}$ of $\mathbb{C}$ respectively. (In fact, sometimes it is convenient to apply $-\log$ and sometimes the identity map, instead of log. We use the notation $h$ as a shorthand for whichever of the three possibilities is appropriate.)

We provide $M_{n}$ with a basepoint $\omega_{x, n}$, whose horizontal coordinate $x$ is large and positive, and which lies in the centre of $M_{n}$. Theorem 7.8 shows that the pair $\left(M_{n}, \omega_{x, n}\right)$ can be mapped by a similarity $z \mapsto a z+b$, where $a>0$, to a very close approximation to the pair $\left(S_{1}, 0\right)$.

In our analysis it is important to distinguish two distinct types of bending line near $p$. This is done in $\S 8$. Let $\sigma \subset \partial \Omega$ be a connected neighbourhood of $p$. A bending line near $p$ has its endpoints in the same component of $\sigma \backslash\{p\}$ or in different components. In the former case, the bending line is called a fringe bending line. We prove results about their shape and position. The other type of bending line is called an arch.

In Lemma 8.4, we prove that the nearest point retraction has dilatation near 1, provided we are sufficiently close to $p \in \partial \Omega$ and sufficiently near the boundary, as measured in $M$. The map $\psi: S_{1} \rightarrow S_{u}$ is obtained from the nearest point retraction $r: \Omega \rightarrow$ Dome $(\Omega)$ by composing with conformal isomorphisms. It therefore has the 
same dilatation as $r$. So properties required of $\psi$ can be deduced from analogous properties of $r$.

In $\S 9$, we define foliations of $\Omega, \Omega_{n}$ and their domes. The leaves of the foliations of the domes are geodesics, and the nearest point retraction induces a map between foliations on the floor and the dome. More precisely, the foliations are defined only near $p$. These foliations will help us to compute partial derivatives and hence dilatations.

\section{Standard examples}

We start the real work with a discussion of some standard examples: the wedge, the half-plane (the wedge with angle $\pi$ ) and the infinite strip, and some special homeomorphisms $\Omega \rightarrow \operatorname{Dome}(\Omega)$ in these standard cases. The particular homeomorphisms that interest us most are the nearest point retraction and the extremal quasiconformal homeomorphism which extends to the identity on $\partial \Omega$. This extremal happens to be unique for these special cases. We use the wedge

$$
U_{\alpha}=\{z:-\alpha / 2<\arg (z)<\alpha / 2\} .
$$

The construction of the dome of a wedge is illustrated in Figures 3.0.i and 3.0.ii. The following lemma is proved by a simple calculation.

3.1. Lemma. The space Dome $\left(U_{\alpha}\right)$ is a half-cone in $\mathbb{U}^{3}$. The nearest point retraction $r: U_{\alpha} \rightarrow$ Dome $\left(U_{\alpha}\right)$ is a real analytic diffeomorphism. Let

$$
u=s(\cos (\alpha / 2), \sin (\alpha / 2) \cos (\theta), \sin (\alpha / 2) \sin (\theta)) \in \text { Dome }\left(U_{\alpha}\right),
$$

where $s>0$ and $0<\theta<\pi$. Then the dilatation at $u$ of the inverse of the nearest point retraction is $1+\sin (\theta) \cos (\alpha / 2)$.

In the case of the infinite strip, which we assume has width 2 , the nearest point retraction is again a real analytic diffeomorphism. We take $u=(s, \cos (\theta), \sin (\theta))$. The dilatation at $u$ of the inverse of the nearest point retraction is $1+\sin (\theta)$.

The dilatation of the nearest point retraction $r: \Omega \rightarrow$ Dome $(\Omega)$, when $\Omega$ is a convex wedge or an infinite strip, is at most 2.

Proof. Using hyperbolic isometries of $\mathbb{U}^{3}$ which preserve the domain and its dome, we can restrict to a single bending line $\lambda$ and its inverse image under the
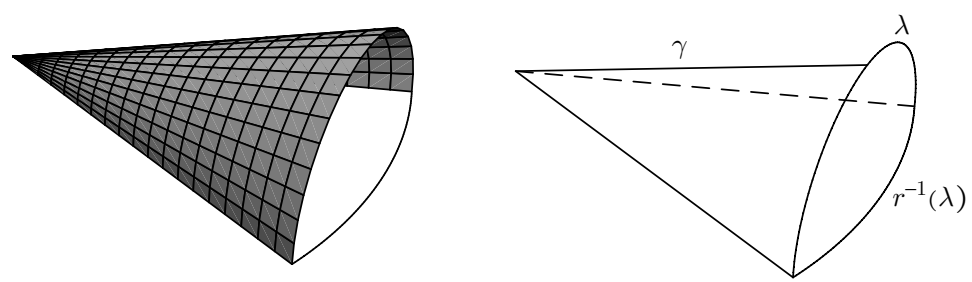

Figure 3.0.i. The dome of a wedge is a cone. In the left-hand picture, the mesh shown consists, on the one hand, of semicircles orthogonal to the plane $\{z=0\}$ and, on the other hand, rays through the origin. The semicircles are hyperbolic geodesics in $\mathbb{H}^{3}$. The rays are not geodesics in $\mathbb{H}^{3}$. However, the ray $\gamma$, running along the highest points of the semicircles, is a geodesic for the induced Riemannian metric on the dome. One of the semicircles is labelled $\lambda$, in agreement with the surrounding text, and its inverse image $r^{-1}(\lambda)$ under the nearest point retraction $r$ is also labelled. 


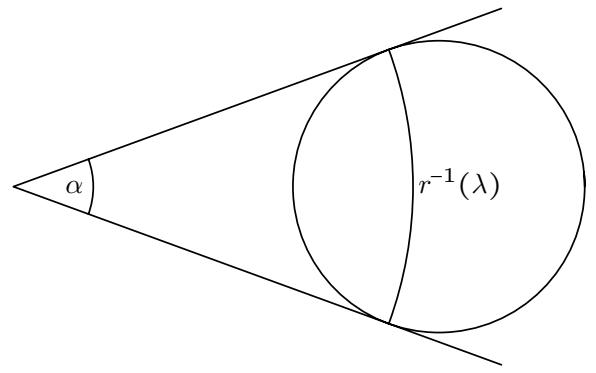

Figure 3.0.ii. This is a picture of the wedge whose dome is shown in Figure 3.0.i. The circle drawn is a circle that is maximal in the wedge. It is the boundary of the hyperbolic plane which contains the geodesic $\lambda$ and is tangent to the cone surface. This hyperbolic plane is a hemisphere. The diagram has been rescaled so that the corresponding sphere has radius 1 . We choose coordinates so that the wedge vertex is the origin. Then $S=(\cot (\alpha / 2), 0,-1)$ is the lowest point of that corresponding sphere. The nearest point retraction $r: r^{-1}(\lambda) \rightarrow \lambda$ is given by radial projection from $S$.

nearest point retraction $r$. Let $u$ and $v$ be the endpoints of $\lambda$. Then $r^{-1}(\lambda)$ is an arc of a circle centred at the vertex of the wedge. The maximal disk $D \subset U_{\alpha}$, with $u, v \in \partial D$, has its centre $c_{0}$ at the intersection of the perpendiculars to $\partial U_{\alpha}$ at $u$ and $v$. We may assume that $D$ has radius 1 . We can compute the coordinates of $c_{0}$ in terms of $\alpha$. Let $p_{0}=c_{0}-(0,0,1)$. Then radial projection from $p_{0}$ sends $r^{-1}(\lambda)$ to $\lambda$ and, on this set is equal to $r$. (To see this, recall that the nearest point retraction $D \rightarrow$ Dome $(D)$ is given by radial projection from $p_{0}$.)

The details of the computation are left to the reader.

Infinite strips play a fundamental role in our proof of Theorem 7.1. To fix the notation, we make the following definition.

3.2. Definition. For each $u>0$, we set $S_{u}=\{z:-u / 2<\operatorname{Im}(z)<u / 2\}$, the infinite horizontal strip of height $u$.

When constructing quasiconformal homeomorphisms $\Omega \rightarrow$ Dome $(\Omega)$, it is much more convenient to construct instead quasiconformal homeomorphisms from one infinite strip to another. The reason this is more convenient is that we can then work with standard concepts involving two real variables, such as partial derivatives; this is much easier than trying to construct and work with the corresponding concepts on a convex hull boundary. To justify this transition to maps between strips, we need to construct conformal isomorphisms between $\Omega$ and an infinite strip, and also between Dome $(\Omega)$ and another infinite strip.

The construction for general convex $\Omega$ will need in-depth investigation. Here we construct these conformal isomorphisms only for the special cases of a wedge or infinite strip. This will give us a useful introduction to the properties applying to general convex subsets $\Omega$.

3.3. Definition. If $\alpha>0$ and we are concentrating on the boundary point $p=0 \in \partial \Omega \subset \mathbb{S}^{2}$ of the wedge $\Omega=U_{\alpha} \subset \mathbb{C}$, we set $h=-\log : U_{\alpha} \rightarrow S_{\alpha}$. Here $S_{\alpha}$ is the infinite horizontal strip of height $\alpha$. If $\alpha>0$ and we are concentrating 
on the boundary point $p=\infty \in \partial \Omega \subset \mathbb{S}^{2}$ of the wedge $\Omega=U_{\alpha} \subset \mathbb{C}$, we set $h=\log : U_{\alpha} \rightarrow S_{\alpha}$. If $\alpha=0$, so that $\Omega=S_{v}$ is an infinite strip for some $v>0$, we set $h: S_{v} \rightarrow S_{v}$ equal to the identity, and take $p=+\infty \subset \partial S_{v} \subset \mathbb{S}^{2}$ to be the boundary point of interest. We set $M$ to be the image of $h$, so that $M=S_{\alpha}$ or $M=S_{v}$, and $h: \Omega \rightarrow M$. In each case, $h$ maps $p \in \partial \Omega$ to $+\infty \in \partial M \subset \mathbb{S}^{2}$.

In order to tie the choice of conformal isomorphism with an infinite strip more tightly to the geometry, and in order to make stronger the parallel with later more general constructions, we proceed as follows. We fix a bending line $\beta_{0}$ in Dome $(\Omega)$, and set $x_{0}$ to be the euclidean midpoint of this semicircular arc in $\mathbb{U}^{3} \subset \mathbb{R}^{3}$. We choose $z_{0} \in \Omega$ so that $r\left(z_{0}\right)=x_{0}$. Let $g: M \rightarrow S_{1}$ be the translation plus change of scale sending $h\left(z_{0}\right) \in M$ to $0 \in S_{1}$.

Next we specify a conformal isomorphism of Dome $(\Omega)$ with an infinite strip. We set $\gamma$ to be the locus of the euclidean midpoints of the bending lines. Since $\Omega$ is a wedge or an infinite strip, this curve is a hyperbolic geodesic with respect to the hyperbolic path metric on Dome $(\Omega)$. We set $u=u(\alpha)=\pi \sin (\alpha / 2) / \alpha$. Let $\iota:$ Dome $(\Omega) \rightarrow S_{u}$ be the isometry of hyperbolic metrics which sends $p$ to $+\infty$ and sends $x_{0} \in$ Dome $(\Omega)$ to $0 \in S_{u}$. Then $\iota(\gamma)$ is the unique horizontal hyperbolic geodesic in $S_{u}$.

We define $\Psi: S_{1} \rightarrow S_{u}$ as the composite

$$
S_{1} \stackrel{g^{-1}}{\longrightarrow} M \stackrel{h^{-1}}{\longrightarrow} \Omega \stackrel{r}{\longrightarrow} \text { Dome }(\Omega) \stackrel{\iota}{\longrightarrow} S_{u} .
$$

We call $\Psi$ the model. We will use it for purposes of comparison with other maps, similarly defined, but using a general convex region $\Omega \subset \mathbb{C}$ with boundary point $p \in \partial \Omega \subset \mathbb{S}^{2}$, instead of a wedge or an infinite strip.

Note that, in the case of a wedge of angle $\alpha$ or an infinite strip

$$
\Psi(t, s)=\left(t,-u / 2+f_{\alpha}\left(\frac{1}{2}+s\right)\right),
$$

where $s \in\left(-\frac{1}{2}, \frac{1}{2}\right)$ and $t \in \mathbb{R}$. The important function $f_{\alpha}:(0,1) \rightarrow(0, u)$ is defined by this equation.

We will need to prove a number of properties of $f_{\alpha}$. This can be done either by direct calculation or by using the method of 'zooming in'. The second method will give less information, though still sufficient for nearly all our purposes in this paper. In view of its general importance, and its ease of use, we now explain this method in the present context.

\section{Parametrized families of surfaces}

The equation

$$
y^{2}+z^{2}=\lambda^{2} x^{2}
$$

defines a cone surface in $\mathbb{R}^{3}$.

We consider a sequence of points $\left(u_{n}\right)_{n \in \mathbb{N}}$ in the plane $z=0$, converging to a point on the cone (not to $(0,0,0))$. We apply a change of scale and translation, so that $u_{n}$ becomes the origin, and the distance of $u_{n}$ from the cone is 1 . This transforms the equation of the cone to a form where it depends real analytically on a certain parameter. Setting this parameter equal to zero, we obtain the equation of a vertical plane. 
In psychological terms, imagine yourself moving towards the cone surface, while you shrink in size at the same rate. The cone surface towers above you, looking more and more vertical as you approach. In the limit the surface becomes a vertical half-plane.

Here is a version of the process in formulas. Let $u(\mu)=(1,-\lambda+\mu, 0)$ be our varying point $u$. We define the similarity $A_{\mu}: \mathbb{R}^{3} \rightarrow \mathbb{R}^{3}$ by $A_{\mu}(v)=\mu v+u(\mu)$. Qualitatively, $A_{\mu}$ has the effect we require. With respect to the new coordinates given by $A_{\mu}$, the cone surface has the equation

$$
-2(1+y) \lambda+\left(1+2 y+y^{2}+z^{2}\right) \mu-x \lambda^{2}(2+x \mu)=0 .
$$

Setting $\mu=0$, we obtain the equation $1+y+\lambda x=0$ of a vertical plane.

Equation (4.0.c) makes rigorous the statement that a family of cone surfaces converges analytically to a vertical plane. We make all objects of interest, for example Riemannian metrics, nearest point retractions, etc., depend analytically on $\mu$. The analytic continuation of the nearest point retraction as $\mu$ changes sign is no longer a nearest point retraction. However, the map itself continues to be defined and meaningful, and it depends analytically on $\mu$.

In a similar way, we can see that various objects vary analytically in the angle $\alpha$ as it moves through 0 to negative values or through $\pi$. When working in this way, we have to be careful to rethink the meaning of the objects, using analysis rather than particular accidents of the English language chosen to describe the objects. For example, there is absolutely no reason why the description 'nearest point retraction' should continue to be a valid description when analytic continuation takes one beyond the original geometric framework in which the nearest point retraction was originally defined.

This method is called 'rescaling' or 'zooming in'.

4.1. Lemma. Let $f$ be the function of equation (3.3.b). There is an $\epsilon>0$ such that $f_{\alpha}(s)$ is the restriction of an analytic function of $\alpha$ and $s$, defined if $|s|<\epsilon$ and $|\alpha|<\pi$. (In fact, we can take $\epsilon=\frac{1}{2}$, but we do not need this.) Also $f_{\alpha}(s)=s+O\left(s^{2}\right)$.

Proof. We have explicit formulas for the conformal isomorphisms $h^{-1} \circ g^{-1}$ : $S_{1} \rightarrow U_{\alpha}$ and $\iota:$ Dome $\left(U_{\alpha}\right) \rightarrow S_{u}$. We are left with proving appropriate analyticity for the nearest point retraction $r: U_{\alpha} \rightarrow$ Dome $\left(U_{\alpha}\right)$. Analyticity in $s$ and $\alpha$ can be proved using the considerations of $\S 4$.

To see that $f(s)=s+O\left(s^{2}\right)$, we use the formula for the nearest point retraction of a half-plane onto a vertical half-plane. We can then apply equation (4.0.c) to see that a similar formula must hold for a cone.

Alternatively, note that the nearest point retraction approximately preserves euclidean distances from $\partial U_{\alpha}$, for points very near $\partial U_{\alpha}$ and far from the vertex of $U_{\alpha}$.

4.2. Remark. Although detailed information about $f_{\alpha}=f$ is not needed in this paper, we provide it in case readers are interested, and also as a check on the above reasoning. We calculate, along the lines indicated in Lemma 3.1, obtaining

$$
\tan \left(\frac{f_{\alpha}(s) \alpha}{\sin (\alpha / 2)}\right)=\frac{-\cos (\alpha / 2)+\cos (\alpha / 2-s \alpha)}{\sin (\alpha / 2) \sin (\alpha / 2-s \alpha)} .
$$


This gives

$$
f_{\alpha}(s)=s+\frac{\alpha}{2 \tan (\alpha / 2)} s^{2}+O\left(s^{3}\right) .
$$

We recall Definition 3.3.a, where the model $\Psi$ is defined. Let $t \in \mathbb{R}$ and $s \in$ $(0,1)$. The dilatation of $\Psi$ at $(t, s) \in S_{1}$ is $f_{\alpha}^{\prime}(s)$. Since the other maps involved in the definition of $\Psi$ are conformal, this is also the dilatation of the inverse of $r$, the nearest point retraction, at the appropriate point. This is recorded in Lemma 3.1 as being equal to $1+\sin (\theta) \cos (\alpha / 2)$, where $\theta$ is the angular coordinate, on a certain vertical semicircle, of the point corresponding to $(s, t) \in S_{1}$. We see that $\theta=f_{\alpha}(s) \alpha / \sin (\alpha / 2)$ and so

$$
f_{\alpha}^{\prime}(s)=1+\sin (\theta) \cos (\alpha / 2)=1+\sin \left(\frac{f_{\alpha}(s) \alpha}{\sin (\alpha / 2)}\right) \cos (\alpha / 2)=1+O(s) .
$$

By comparing Lemma 3.1 with the next result, we will see that the nearest point retraction does not give an extremal quasiconformal homeomorphism.

4.3. Lemma. With respect to the euclidean path metric, Dome $\left(U_{\alpha}\right)$ is isometric to a wedge of angle $\beta=\pi \sin (\alpha / 2)$. There is a unique extremal quasiconformal homeomorphism between this wedge and the wedge $U_{\alpha}$, corresponding to the identity map on $\partial U_{\alpha}$, namely the homeomorphism that preserves euclidean distance from the vertex and scales linearly the angle subtended at the vertex. The dilatation is constant and is equal to $\beta / \alpha$.

Proof. The logarithm function maps the domain $U_{\alpha}$ conformally to $S_{\alpha}$ (for the meaning of $S_{\alpha}$, see Definition 3.2). Now Dome $\left(U_{\alpha}\right)$, which, with the euclidean path metric is euclidean isometric to a wedge of angle $\beta$, is mapped by the logarithm to $S_{\beta}$. We have a quasiconformal homeomorphism $q: S_{\alpha} \rightarrow S_{\beta}$, given by $q(x, y)=$ $(x, \beta y / \alpha)$. The restriction of $q$ to $\partial S_{\alpha}$ is the obvious map corresponding to the identity on $\partial U_{\alpha}$.

The corresponding quasiconformal homeomorphism, still denoted by $q$ but now a map $q: S_{1} \rightarrow S_{\beta / \alpha}$, is given by the same formula $q(x, y)=(x, \beta y / \alpha)$. It is better to divide by $\alpha$ because the new maps are applicable also when $\alpha=0$, in which case $U_{\alpha}$ can be interpreted as an infinite strip, and $\beta / \alpha$ as $\pi / 2$.

To prove our lemma, we apply a result due to Strebel and, independently, to Beurling and Ahlfors, which states that, given an affine map $A: \mathbb{R}^{2} \rightarrow \mathbb{R}^{2}$, such that $A\left(S_{1}\right)=S_{u}$, there is a unique extremal quasiconformal map $q: S_{1} \rightarrow S_{u}$ among homeomorphisms $f: S_{1} \rightarrow S_{u}$ such that $f\left|\partial S_{1}=A\right| \partial S_{1}$, namely $q=A \mid S_{1}$. An easy proof, if one requires to know only the extremality, which is all that is required in this paper, and not the uniqueness, is provided in $[\mathbf{3}, \S 3]$.

The above proof also works for $\alpha=\pi$, when the nearest point retraction $r$ is the relevant extremal quasiconformal mapping, and the dilatation is 1 . We have also proved the following result.

4.4. Corollary. With respect to the euclidean path metric, Dome $\left(S_{1}\right)$ is isometric to the strip $S_{\pi / 2}$. There is a unique extremal quasiconformal homeomorphism $q: S_{1} \rightarrow S_{\pi / 2}$ corresponding to the identity map on $\partial S_{1}$, given by $q(x, y)=(x, \pi y / 2)$. The dilatation is constant and is equal to $\pi / 2$. 
4.5. The general convex region $\Omega \subset \mathbb{C}$

4.6. TheOREM. The Thurston isometry $\iota: \mathbb{H}^{2} \rightarrow$ Dome $(\Omega)$ is a proper $C^{1}$-map with derivative everywhere of rank 2 , and Dome $(\Omega)$ is a proper $C^{1}$-submanifold of $\mathbb{U}^{3}$.

4.7. REMARK. Although the dome is differentiable, the nearest point retraction $r: \Omega \rightarrow$ Dome $(\Omega)$ may not be differentiable. In fact, it is unusual to have a convex region $\Omega$ for which the nearest point retraction is everywhere differentiable. Since quasiconformal maps are differentiable almost everywhere, one can however deduce from Theorem 5.1 that $r$ is differentiable almost everywhere. A related point is that the dome of a convex region is usually not a $C^{2}$-submanifold of $\mathbb{U}^{3}$.

Before proving Theorem 4.6, we establish some general properties of the bending lamination for the dome of a convex region.

4.8. Lemma. Let $x \in \partial \Omega$ be the endpoint of at least one bending line. (The boundary of $\Omega$ is taken in $\mathbb{S}^{2}$.) Then the following must be true:

(4.8.1) $x \neq \infty$;

(4.8.2) $\partial \Omega$ has a unique tangent at $x$;

(4.8.3) Dome $(\Omega)$ has no isolated bending line and each bending line has zero transverse measure;

(4.8.4) the nearest point retraction $r: \Omega \rightarrow$ Dome $(\Omega)$ is a homeomorphism;

(4.8.5) there are at most two bending lines with $x$ as endpoint; if $x$ is the endpoint of two distinct bending lines, then there is a flat such that each of the two bending lines is a boundary component of the flat (see Figure 4.8).

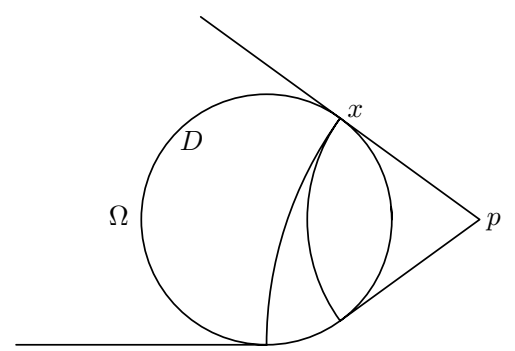

Figure 4.8. This illustrates (4.8.5). We show the convex set $\Omega$ and a maximal disk $D \subset \Omega$. Two bending lines for Dome $(\Omega)$ have a common endpoint $x$. The bending lines are not shown. Instead we show their inverse images under the nearest point retraction.

Proof. First we deal with the case $x=\infty$. Since $\Omega$ is euclidean convex, a maximal disk in $\Omega$ can have $\infty$ in its closure only if it is equal to $\Omega$. But then there are no bending lines and $x$ cannot exist. For the remainder of the proof, we assume that $x$ is finite.

If a bending line has positive transverse measure, then $\partial \Omega$ has a corner at the endpoint $x$, and the bending is in the wrong direction, preventing $\Omega$ from being convex. The first three conclusions follow.

In order to prove (4.8.5), suppose $x$ is an endpoint of a bending line $\ell$. Let $P$ be a support plane for $\Omega$ which contains $\ell$. There is a corresponding maximal disk $D \subset \Omega$, such that $x \in \partial D=\partial P$. Let $y$ be the other endpoint of $\ell$. Then $y \in \partial D$. 
Since the tangent line to $\partial \Omega$ at $x$ is unique, there is exactly one maximal disk $D \subset \Omega$ such that $x \in \partial D$. It follows that any bending line with $x$ as an endpoint must lie in $P$. Moreover, $P \cap \operatorname{Dome}(\Omega)$ is the convex hull of $\partial \Omega \cap \partial D$. The statement (4.8.5) now follows. The situation is illustrated in Figure 4.8.

Proof of Theorem 4.6. Let $u \in$ Dome $(\Omega)$. If $u$ lies in a flat, then there is clearly a smooth chart. If $u$ lies on a bending line, we see from Lemma 4.8 that the Thurston isometry is $C^{1}$.

\section{Quasiconformality of the nearest point retraction}

5.1. TheOREm. Let $\Omega$ be a proper open convex subset of $\mathbb{C}$, possibly with noncompact closure, and let $r: \Omega \rightarrow$ Dome $(\Omega)$ be the nearest point retraction. Then $r$ is a 2-quasiconformal homeomorphism.

To prove Theorem 5.1, we will use approximation theorems from [3]. For the main result of this chapter (Theorem 7.1), we will rely heavily on Theorem 5.2, used in the current paper only with the additional (and unnecessary) restriction that $\Omega$ and $\Omega_{n}$ are all convex.

5.2. Theorem (The Approximation Theorem). Let $K \geqslant 1$, and let $\left(\Omega_{n}\right)_{n \in \mathbb{N}} \subset$ $\mathbb{C}$ be a sequence of simply connected proper open subsets converging to a simply connected proper open subset $\Omega \subset \mathbb{C}$. (The convergence is Hausdorff convergence of the compact complements in $\mathbb{S}^{2}$.) Given any sequence

$$
\left(\phi_{n}: \Omega_{n} \rightarrow \text { Dome }\left(\Omega_{n}\right) \subset \overline{\mathbb{D}^{3}}\right)_{n \in \mathbb{N}}
$$

of $K$-quasiconformal homeomorphisms which extend continuously to the identity on the common boundary, there is a subsequence, converging uniformly on compact subsets, to a $K$-quasiconformal $\phi: \Omega \rightarrow$ Dome $(\Omega) \subset \overline{\mathbb{D}^{3}}$ which extends continuously to the identity on the common boundary.

In order to prove Theorem 5.1, we will not rely on Theorem 5.2, but only on the more easily proved Theorem 5.3. This result, not stated explicitly in [3], is implicitly contained in the proof of $[\mathbf{3}$, Lemma 7.5$]$. Alternatively, if we assume $\Omega$ and $\Omega_{n}$ to be convex, which is all that we need in the present paper, then it is easy to deduce Theorem 5.3 from Theorem 5.2, which is explicitly stated and proved in [3]. Although we need only the convex case, the more general statement given in Theorem 5.3 is true for any $\Omega$ and $\Omega_{n}$.

5.3. TheOREm. Let $\left(\Omega_{k}\right)_{k \in \mathbb{N}}$ be a sequence of open simply-connected regions in $\mathbb{S}^{2}$ converging to the simply-connected region $\Omega$, in the sense of Hausdorff convergence of their compact complements in $\mathbb{S}^{2}$. Let $r_{k}: \Omega_{k} \rightarrow \operatorname{Dome}\left(\Omega_{k}\right)$ and $r: \Omega \rightarrow$ Dome $(\Omega)$ be the nearest point retractions. Let $\iota_{k}:$ Dome $\left(\Omega_{k}\right) \rightarrow \mathbb{H}^{2}$ and $\iota:$ Dome $(\Omega) \rightarrow \mathbb{H}^{2}$ be the Thurston isometries, normalized consistently in some sensible way (as explained in $[\mathbf{3}, \S 7.8])$. Then the sequence $\left(\iota_{k} \circ r_{k}\right)_{k \in \mathbb{N}}$ converges to $\iota \circ r$ uniformly on compact subsets of $\Omega$.

Proof of Theorem 5.1. By the above approximation theorems, we need only prove that $r$ is 2-quasiconformal when $\Omega$ is a finite-sided convex euclidean polygon, 
possibly with non-compact closure. Let $V$ be a flat or a bending line in Dome $(\Omega)$. If $V$ is a flat, then $r$ is conformal on $r^{-1}(V)$. Otherwise $V$ is a geodesic joining two of the edges of the polygon $\Omega$. Note that $V$ cannot end at a vertex of the polygon. We denote these two edges of the polygon by $\sigma_{1}$ and $\sigma_{2}$. The union of bending lines with one endpoint in $\sigma_{1}$ and the other endpoint in $\sigma_{2}$ is a closed subset of Dome $(\Omega)$. By (4.8.2), none of the four points which are endpoints of the intervals $\sigma_{1}$ or $\sigma_{2}$ can be the endpoints of any bending line. See Figure 5.3.

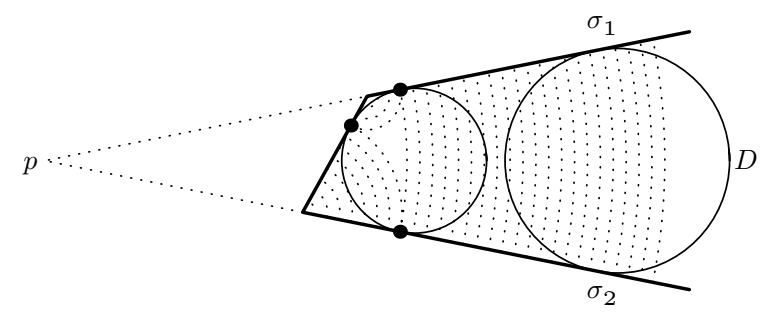

Figure 5.3. This illustrates the proof of Theorem 5.1. The boundary of the polygon is indicated by thick lines. The edges $\sigma_{1}$ and $\sigma_{2}$, when extended, meet at $p$. The letter $D$ denotes the maximal disk $D$ in the proof. In the diagram we show two possible positions for $D$. The rest of this caption refers only to the extreme position of $D$ towards the left. The geodesic $V$ is the last bending line joining $\sigma_{1}$ to $\sigma_{2}$. The hyperbolic plane $P$ with $\partial P=\partial D$ is divided into two halves by $V$. The left half of $P$ meets Dome $(\Omega)$ and the right half does not meet it. This is the basis of the general reasoning which shows that we can move $D$ a little to the right by scaling with centre $p$, while retaining the property that it is a maximal disk of the polygon $\Omega$, tangent both to $\sigma_{1}$ and to $\sigma_{2}$.

Suppose that, when extended, $\sigma_{1}$ and $\sigma_{2}$ meet at a point $p$. (If the sides are parallel, they meet at infinity. We leave to the reader the obvious interpretation of the following remarks in this case.) Let $D \subset \Omega$ be the maximal disk such that the endpoints of $V$ lie on $\partial D$. Let $P$ be the hyperbolic plane with $\partial P=\partial D$. Then $P \cap$ Dome $(\Omega)$ is either equal to $V$ or to an ideal hyperbolic polygon, one of whose sides is $V$. Therefore at least one component of $P \backslash V$ in $P$ does not meet Dome $(\Omega)$. It follows that scaling by positive real numbers either in $(1-\epsilon, 1]$ or by positive real numbers in $[1,1+\epsilon)$, with $p$ as fixed point, will move $D$ through maximal disks in $\Omega$ to which both $\sigma_{1}$ and $\sigma_{2}$ are tangent.

So the subset of Dome $(\Omega)$ consisting of bending lines with endpoints on $\sigma_{1}$ and $\sigma_{2}$ has non-empty interior. The computation of the dilatation of $r$ can therefore be reduced to the computation for such a subset. This can be carried out by extending $\sigma_{1}$ and $\sigma_{2}$ until they meet, bounding a wedge of angle $\alpha$ with vertex $p$. By Lemma 3.1, the dilatation lies between 1 and $1+\cos (\alpha / 2) \leqslant 2$.

\section{Boundary dilatation}

We have shown above that, for a finite-sided convex region, the dome consists of a finite number of pieces, each of which is either flat or part of a cone surface. Now we return to the case of a general open convex region in the plane.

6.1. Definition. For each point $p \in \partial \Omega$, we have a minimal wedge $W_{p} \supset \Omega$, with vertex at $p$. We set $\operatorname{ang}(p)$ equal to the angle of $W_{p}$. We define the directed tangent lines at $p$ to $\Omega$ to be the two lines containing the two edges of $W_{p}$. We direct 
the two tangent lines so that they point in a counterclockwise direction around $\Omega$. See the picture in Figure 6.1.

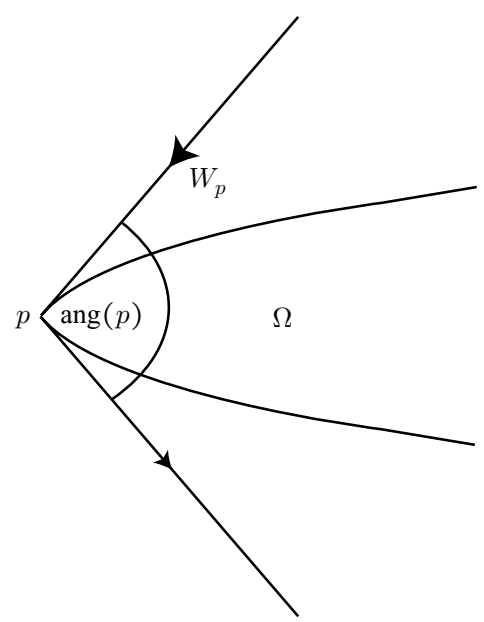

Figure 6.1. This illustrates Definition 6.1. The region $W_{p}$ is the wedge contained between the two tangents to $\partial \Omega$ at $p$. We have $\Omega \subset W_{p}$.

The angle between the two directed tangent lines is equal to $\pi-\operatorname{ang}(p)$. If $\operatorname{ang}(p)=\pi$, then the two directed tangent lines are equal.

The following lemma is obvious.

6.2. Lemma. Let $\left(p_{i}\right)_{i \in \mathbb{N}}$ be a sequence in $\partial \Omega$ converging to $p$ from one side of $p$. For each $i$, let $\tau_{i}$ be either of the two directed tangent lines at $p_{i}$. Then $\left(\tau_{i}\right)_{i \in \mathbb{N}}$ converges to $\tau$, the directed tangent line at $p$ on the same side of $p$ as $\left(p_{i}\right)_{i \in \mathbb{N}}$.

6.3. Definition. We extend Definition 6.1 to the case $p=\infty$ as follows. We fix a point $q \in \partial \Omega$ and define $W_{\infty, q} \subset \Omega$ to be the closure of the maximal wedge with vertices at $q$ and $\infty$ contained in $\Omega$. We define $\operatorname{ang}(\infty)$ to be the angle of $W_{\infty, q}$. It is easy to see that this angle is independent of $q$. See the picture in Figure 6.3.

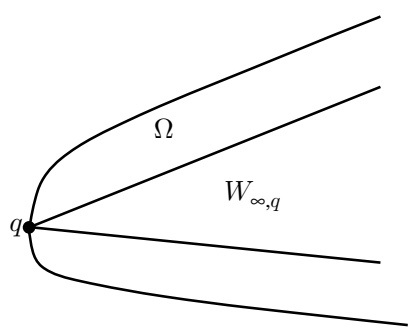

Figure 6.3. This illustrates Definition 6.3. We have $q \in \partial \Omega$. The region $W_{\infty, q} \subset \Omega$ is the wedge defined in Definition 6.3.

Note that $W_{\infty, q} \subset \Omega$ may be degenerate, collapsing to a single line. Whether it is degenerate or not, we obtain two directed tangent lines to $\partial \Omega$ at $\infty \in \mathbb{S}^{2}$. We have $0 \leqslant \operatorname{ang}(\infty) \leqslant \pi$, where 0 corresponds to the degenerate case.

We define $0 \leqslant \operatorname{ang}_{0} \leqslant \pi$ to be the minimum of $\operatorname{ang}(p)$, as $p$ varies over $\partial \Omega \subset \mathbb{S}^{2}$. 
6.4. Example. An example where $\operatorname{ang}(\infty)=0$ is given by the set

$$
\left\{(x, y): y^{2}<x\right\} \text {. }
$$

This is one component of the complement of a parabola.

6.5. Remark. If $p=\infty$ and $\operatorname{ang}(p)=\pi$, then it is easy to see that $\Omega$ is a half-plane.

Let $U$ and $V$ be proper open simply connected subsets of the plane. Let $h_{U}: U \rightarrow \mathbb{D}^{2}$ and $h_{V}: V \rightarrow \mathbb{D}^{2}$ be Riemann mappings. Two quasiconformal homeomorphisms $f_{1}, f_{2}: U \rightarrow V$, are said to have the same boundary values if $h_{V} f_{1} h_{U}^{-1}, h_{V} f_{2} h_{U}^{-1}: \mathbb{D}^{2} \rightarrow \mathbb{D}^{2}$ have the same boundary values. (Recall that a quasiconformal homeomorphism between two open unit disks can always be continuously extended to the closed disks, so the 'boundary values' of these quasiconformal homeomorphisms makes sense.)

6.6. Definition. Given a quasiconformal homeomorphism $f: U \rightarrow V$ and a point $p \in \partial U$, the notion of substantial boundary dilatation $H_{p}(f)$ of $f$ at $p$ is defined as follows. Denote the disk of radius $s$ about $p$ by $B_{s}(p)$. Suppose we have a quasiconformal map $g: U \rightarrow V$ with the same boundary values as $f$ in the sense just described (write $g \sim f$ ). We denote the maximal dilatation of $g$ restricted to $B_{s}(p) \cap U$ by $K_{s}(g)$. We make the following sequence of definitions:

$$
\widetilde{H_{p}}(g)=\lim _{s \searrow 0} K_{s}(g), \quad H_{p}(f)=\inf _{g \sim f} \widetilde{H_{p}}(g), \quad H(f)=\max _{p \in \partial U} H_{p}(f) .
$$

The number $H(f)$ is called the boundary dilatation of $f$. It is known $[\mathbf{4}, \mathbf{5}]$ that the maximum is attained for some $p \in \partial U$.

Automatically $H(f) \leqslant K_{0}$ where $K_{0}$ is the maximal dilatation of an extremal quasiconformal map with the boundary values of $f$. Typically, there is strict inequality. See [7] for an exposition of this topic.

\section{The main theorem}

7.1. Theorem. Let $r: \Omega \rightarrow$ Dome $(\Omega)$ be the nearest point retraction. If $\operatorname{ang}(p)>0$, then the substantial boundary dilatation of $r$ at $p$ is given by

$$
H_{p}(r)=\pi \sin (\operatorname{ang}(p) / 2) / \operatorname{ang}(p) .
$$

If $\operatorname{ang}(p)=\pi$, then $H_{p}(r)=1$. If $\operatorname{ang}(p)=0$, then $H_{p}(r)=\pi / 2$. The boundary dilatation is obtained by substituting $\operatorname{ang}_{0}$ for $\operatorname{ang}(p)$ in these formulas (see Definition 6.3).

The proof will occupy the remainder of this paper.

\section{2. $\quad$ Proof for special cases}

Suppose the boundary in $\mathbb{S}^{2}$ of the convex open subset $\Omega \subset \mathbb{C}$ includes $\infty$. The closure $\bar{\Omega}$ of $\Omega$ in $\mathbb{C}$ then has one or two ends. If there are two, then $\Omega$ must be an infinite strip. In this case, Lemma 4.3 is much stronger than Theorem 7.1. So, for 
the remainder of the proof of Theorem 7.1, we assume that $\bar{\Omega}$ has at most one end, which is its boundary point at $\infty$.

Next we think about the case when $\operatorname{ang}(p)=\pi$. We need to prove that the substantial boundary dilatation at $p$ is 1 . If $p=\infty$ and $\operatorname{ang}(p)=\pi$, then, as already pointed out in Remark $6.5, \Omega$ is a half-plane and the nearest point retraction is conformal. This reduces consideration of $\operatorname{ang}(p)=\pi$ to the case when $p$ is finite.

Given $u \in \Omega$ near $p$, we need to show that the dilatation of $r$ near $u$ is not much bigger than 1 . We approximate $\Omega$ by a finite-sided convex polygon, with internal angles near $p$ very close to $\pi$. We complete the proof in this special case following the method of Theorem 5.1. The reader is referred to Figure 5.3 which shows that the dilatation of the nearest point retraction can be computed from the computation for a wedge. Use of the nearest point retraction as the relevant homeomorphism satisfying Theorem 7.1, rather than some specially constructed homeomorphism, is appropriate only because we are near a point with angle $\pi$ so that its dilatation is nearly 1 .

Our conclusions from these few remarks are that, when proving Theorem 7.1, we can confine our attention to points $p \in \partial \Omega$, finite or infinite, such that $0 \leqslant \operatorname{ang}(p)<$ $\pi$. Moreover, if $p=\infty$, we may assume it is the only boundary point at infinity.

Given a convex open region in the plane, its closure in $\mathbb{S}^{2}$ is a closed topological disk, except for an infinite strip. But this is excluded by the previous paragraph. So we assume from now on that the closure of $\Omega$ in $\mathbb{S}^{2}$ is a closed topological disk.

\subsection{Standard position for $\Omega$}

We recall from $\S 7.2$ that we may assume that $\operatorname{ang}(p)<\pi$ and that $\bar{\Omega}$ has at most one end. We will find a similarity that puts $\Omega$ into a convenient position for applying the log function.

First we look at the case $p=\infty$. For each finite $q \in \partial \Omega$, we have a closed wedge (or single ray) $W_{\infty, q} \subset \Omega$ (see Definition 6.3). As $q$ varies, the edges of $W_{\infty, q}$ vary in either one or two parallel families of lines.

It follows from continuity (see Figure 7.3) that we can find a point $q$ with the property that the bisector of $W_{\infty, q}$ is orthogonal to a support line for $\Omega$ at $q$. We apply a similarity to make the support line vertical with $\Omega$ on its right, and move $q$ to 0 .

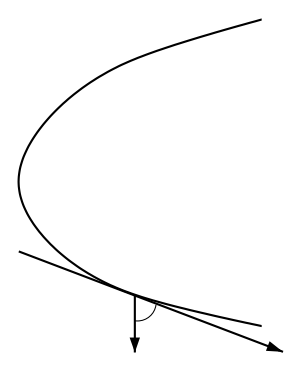

FiguRE 7.3. This illustrates the first paragraph of $\S 7.3$.

We recall that $p$ is the corner point defined in Theorem 7.1. If $p=\infty$, we have defined $q$ and described a normalization, with $q=0$. This normalization fixes a branch of $\log : \Omega \rightarrow \mathbb{C}$.

Now we consider the case where $p$ is finite. Then $\operatorname{ang}(p)>0$. Following Definition 6.1 , we set $W_{0}$ equal to the minimal wedge containing $\Omega$ with vertex 
at $p$. We may assume that $p=0$ and that $W_{0}$ is a wedge with rays in the range $(-\operatorname{ang}(p) / 2, \operatorname{ang}(p) / 2)$. Once again, this fixes a branch of $\log : \Omega \rightarrow \mathbb{C}$.

7.4. Definition. (1) If $p=0$, we set $h=-\log$.

(2) If $p=\infty$ and $\operatorname{ang}(\infty)>0$, we set $h=\log$.

(3) If $p=\infty$ and $\operatorname{ang}(\infty)=0$, we set $h$ equal to the identity map.

In each case, we define $M=h(\Omega)$. After $h$ is extended continuously to the closure of $\Omega$ in $\mathbb{S}^{2}, p$ is sent to a point $+\infty \in \partial M$. In each case $M=h(\Omega)$ is somewhat like a horizontal strip. The word 'somewhat' does need to be taken seriously, as we see with Example 6.4, when $p=\infty$, ang $(p)=0$ and $M=\Omega$. The notation reminds us that $+\infty$ is thought of as lying at infinity on the right of $M$.

Since $\Omega$ is convex, $\partial \Omega$ has a well-defined tangent except at a countable set of points, at each of which there are exactly two distinct tangent directions. It follows that $\partial M$ has a well-defined tangent except at a countable set of points, where there are exactly two distinct tangents. Near the boundary point $+\infty$, the tangents to $\partial M$ are almost horizontal.

7.5. Definition. As in Definition 3.3, we fix a bending line $\beta_{0}$ whose image under $\iota$ joins the two components of $\partial S_{u}$. We fix $x_{0}$ to be the euclidean midpoint of the semicircular arc $\beta_{0} \subset$ Dome $(\Omega)$, and set $z_{0}=r^{-1}\left(x_{0}\right) \in \Omega$. Without loss of generality (changing the choice of $\beta_{0}$ ), we may assume that all tangent lines to $\partial M$ to the right of $h\left(z_{0}\right)$ have slope less than 0.001 in absolute value.

To the right of $h\left(z_{0}\right), \partial M$ breaks up into two components: the upper component $\partial_{+} M$ and the lower component $\partial_{-} M$. The corresponding parts of $\partial \Omega$ are denoted by $\partial_{+} \Omega$ and $\partial_{-} \Omega$.

\subsection{Approximating $\Omega$}

Some proofs turn out to be easier when $\Omega$ has a special form, namely when $\partial \Omega \backslash\{p\}$ is the union of a locally finite family of straight edges. For this reason, we will work with an approximation $\Omega_{n}$ to $\Omega$, which has such a locally finite family of edges.

One slightly subtle point is that we make our proofs and estimates using $\Omega_{n}$, but with conclusions which are independent of $n$. To see why we find this necessary, recall that we are computing the substantial boundary dilatation of the nearest point retraction $r: \Omega \rightarrow \operatorname{Dome}(\Omega)$ at $p$ (see Definition 6.6). We need to show that we can find a very small neighbourhood $N$ of $p$ and $\tilde{r}: \Omega \rightarrow \operatorname{Dome}(\Omega)$, with the same boundary values as $r$ and such that $\tilde{r} \mid N$ has dilatation not much larger than $\pi \sin (\operatorname{ang}(p) / 2) / \operatorname{ang}(p)$. We will show that $N$ can be chosen independently of $n$, so that, if $\Omega$ is replaced by $\Omega_{n}$ and $r$ by $r_{n}$, there is an $\tilde{r}_{n}$ with the same boundary values as $r_{n}$, such that $\tilde{r}_{n} \mid N$ has dilatation uniformly near $\pi \sin (\operatorname{ang}(p) / 2) / \operatorname{ang}(p)$. (That is, the closeness of the dilatation does not depend on $n$, but only on N.) As usual, interchanging of two limits (in this case, convergence of $N$ to $p$ and convergence of $n$ to infinity) depends on some kind of uniform behaviour, or independence of the exact value of $n$.

We define $\Omega_{n}$ as the intersection of certain supporting open half-planes of $\Omega$. We specify $\Omega_{n}$ by specifying $X_{n} \subset \partial \Omega$, the set of points at which we take supporting half-planes. If $x \in X_{n}$ is a point with two tangent lines, we use both supporting 

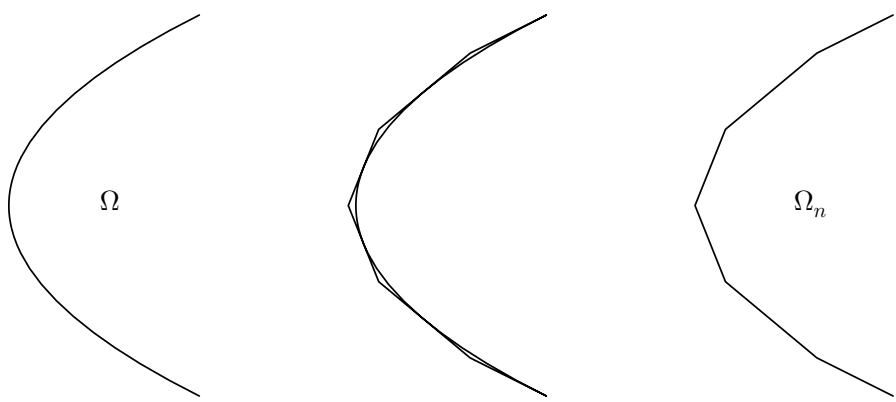

FiguRE 7.6. The left-hand figure is a convex curve, enclosing a convex region $\Omega$. The point $p$ is at infinity on the right. The middle picture shows $\Omega$ together with a number of support lines, as described in $\S$ 7.6. Using the support lines only, we obtain the polygonal approximation $\Omega_{n}$, as shown in the right-hand picture.

half-planes. (Or, if one prefers, one can avoid the countable set of points where $\partial \Omega$ has two distinct tangent lines.) See Figure 7.6.

Suppose we have defined subsets

$$
X_{i} \subset\left(\partial \beta_{0} \cup \partial_{+} \Omega \cup \partial_{-} \Omega\right)
$$

for $1 \leqslant i \leqslant n-1$, so that

$$
\partial \beta_{0} \subset X_{1} \subset \ldots \subset X_{n-1} \subset \partial \Omega \backslash\{p\} .
$$

We define $X_{n}$ so that the following conditions apply.

(7.6.1) $\partial \beta_{0} \subset X_{n}$.

(7.6.2) $X_{n}$ is closed in $\partial \Omega \backslash\{p\}$.

(7.6.3) $X_{n}$ is locally finite.

(7.6.4) $X_{n-1} \subset X_{n}$.

(7.6.5) Each component of $\left(\partial_{-} \Omega \cup \partial_{+} \Omega\right) \backslash X_{n-1}$ containing the endpoint of a bending line joining $\partial_{-} \Omega$ to $\partial_{+} \Omega$, also contains a point of $X_{n}$ that is the endpoint of such a bending line.

(7.6.6) If $x \in X_{n}$ is an endpoint of a bending line joining $\partial_{-} \Omega$ to $\partial_{+} \Omega$, then the other endpoint is also in $X_{n}$. To see that this requirement is consistent with the local finiteness requirement, consider a chain $x_{1}, \ldots, x_{k} \in \partial \Omega$ such that, for each $i \geqslant 1$, the geodesic $x_{i} x_{i+1}$ is a bending line in Dome $(\Omega)$ joining $\partial_{-} \Omega$ to $\partial_{+} \Omega$. By Lemma 4.8.3, such a chain must satisfy $k \leqslant 3$.

(7.6.7) Each point of $\partial_{+} M \cup \partial_{-} M$ is within a distance $2^{-n}$ of the image of $X_{n}$ in $\partial M$.

Each point $x \in X_{n}$ gives rise to either one or two half-planes containing $\Omega$. Their intersection is a convex open region $\Omega_{n} \supset \Omega$ for each $n$, and $\left(\Omega_{n}\right)_{n \in \mathbb{N}}$ converges to $\Omega$. We have $p \in \partial \Omega_{n}$. We set $M_{n}=h\left(\Omega_{n}\right)$, where $h=-\log$ if $p$ is finite and $0<\operatorname{ang}(p)<\pi, h=\log$ if $p=\infty$ and $0<\operatorname{ang}(p)<\pi$, and $h=$ Id if $p=\infty$ and $\operatorname{ang}(p)=0$.

\subsection{Construction of $g$ and $G_{n}$}

We next choose a conformal isomorphism $g: M \rightarrow S_{1}$, such that $g \circ h$ sends the $p \in \partial \Omega$ to the point $+\infty \in \partial S_{1}$. We require that $g\left(h\left(z_{0}\right)\right)=0$, where $z_{0}$ is defined in $\S 7.5$. This completely determines $g$. 
Similarly, we set $M_{n}=h\left(\Omega_{n}\right)$ and define $g_{n}: M_{n} \rightarrow S_{1}$ to be the unique conformal isomorphism such that $g_{n}\left(h\left(z_{0}\right)\right)=0$ and $g_{n} \circ h$ sends $p$ to $+\infty$.

We use the notation $\partial_{-} M_{n}$ and $\partial_{+} M_{n}$, with its obvious meaning. For large $x>0$, set $v_{x, n} \subset M_{n}$ to be the vertical interval with $x$-coordinate equal to $x$, joining $\partial_{-} M_{n}$ to $\partial_{+} M_{n}$. Let $\sigma_{x, n}: \mathbb{C} \rightarrow \mathbb{C}$ be the affine map $z \mapsto a z+b$, sending the interval $(-i / 2, i / 2)$ to $v_{x, n}$. Here $a>0$ is the length of $v_{x, n}$ and $\operatorname{Re}(b)=x$. The imaginary part of $b$ depends on the situation: unless $p=\infty$ and $\operatorname{ang}(p)=0$, the imaginary part of $b$ converges to zero as $x$ tends to infinity. If $p$ is finite, then the length of $v_{x, n}$ increases monotonically as $x$ tends to infinity, with limit $\operatorname{ang}(p)$. If $p=\infty$ and $0<\operatorname{ang}(p)<\pi$, then its length decreases monotonically, with limit $\operatorname{ang}(p)$.

Let $P_{x, n}$ be the inverse image of $M_{n}$ under $\sigma_{x, n}$.

7.8. Theorem. Let $(x(j))_{j \in \mathbb{N}}$ be a sequence of real numbers tending to $+\infty$, and let $(n(j))_{j \in \mathbb{N}}$ be an arbitrary sequence of positive integers. For each $j$, let $\tau_{j}$ be the unique horizontal translation such that the composite, $\rho_{j}$ given by

$$
\rho_{j}: P_{x(j), n(j)} \stackrel{\sigma_{x(j), n(j)}}{\longrightarrow} M_{n(j)} \stackrel{g_{n(j)}}{\longrightarrow} S_{1} \stackrel{\tau_{j}}{\longrightarrow} S_{1},
$$

maps 0 to a point in $S_{1}$ on the $y$-axis. Given a compact subset $C$ of the infinite open strip $S_{1}$ and an $\epsilon>0$, there is a $j_{0}$ such that, if $j \geqslant j_{0}$, then $\left|\rho_{j}-\operatorname{Id}\right|<\epsilon$ on $C$. Moreover, $j_{0}$ can be chosen independently of the sequence $(n(j))_{j \in \mathbb{N}}$.

We can allow one or more of the $n(j)$ above to equal $\infty$. In this case, we replace $\Omega_{n(j)}$ by $\Omega$, and correspondingly for the other terms, such as $M, g, v$ and $\sigma$. Theorem 7.8 continues to be true in this greater generality.

Proof. We apply Caratheodory's Kernel Theorem (see [6, Theorem 1.8]) to the fixed point 0 in the varying region $P_{x(j), n(j)}$. We see that the conformal mapping $\phi_{j}: S_{1} \rightarrow P_{x(j), n(j)}$, fixing 0 , and with positive derivative at 0 , converges to the identity map $S_{1} \rightarrow S_{1}$, uniformly on compact sets. An argument by contradiction shows that the rate of convergence is independent of the choice of the sequence $(n(j))_{j \in \mathbb{N}}$.

The Poincaré metric in $S_{1}$ is $\pi|d z| / \sin (\pi y)$. Therefore the metric in $P_{x(j), n(j)}$ is near this on any compact subset $C \subset S_{1}$, for large $j$. We deduce that any geodesic for the hyperbolic metric on $P_{x(j), n(j)}$ for large $j$, that travels a substantial horizontal distance, must follow close to the central geodesic of $S_{1}$, the line $y=0$, for some (lesser) substantial distance. If one wanted to, one could get numerical estimates through one's knowledge of the shapes of right-angled hyperbolic triangles. It follows from $\S 7.5$ and $\S 7.7$ that the inverse image under $\rho_{j}: P_{x(j), n(j)} \rightarrow S_{1}$ of the central line of $S_{1}$ is a real analytic path that approximates the central line closely in the $C^{\infty}$-topology in any compact subset $C \subset S_{1}$, provided that $j$ is large enough.

The claimed convergence follows. The independence of $j_{0}$ on the sequence $(n(j))_{j \in \mathbb{N}}$ follows by using an argument by contradiction.

We have also proved the following result.

7.9. Corollary. We use the notation of Theorem 7.8. The geodesic in $P_{x(j), n(j)}$ from $\left(\sigma_{x(j), n(j)}\right)^{-1}\left(h\left(z_{0}\right)\right)$ to $+\infty$ approximates the line $y=0$ more and more closely in the $C^{\infty}$-topology in the region $x \geqslant 0$, as $j$ tends to infinity. This convergence is uniform in the sense that it is independent of the sequence $(n(j))_{j \in \mathbb{N}}$. 


\section{Fringe bending lines}

In order to get precise results about the dilatation of the nearest point retraction, we need to study bending lines for $\Omega$ and $\Omega_{n}$ in more detail. As in Figure 8.0, we consider the position of a bending line $\lambda \subset$ Dome $(\Omega)$, such that both endpoints $p_{1}$ and $p_{2}$ of $\lambda$ lie in $\partial_{+} \Omega$ (or equivalently, both in $\partial_{-} \Omega$ ). We take $\lambda$ very near to $p$. Then there is a unique maximal disk $D \subset \Omega$, such that the $p_{1}, p_{2} \in \partial D$. Set $s_{D}$ to be the radius of $D$ and $\theta$ to be the change in the angle of the directed tangent line between the two endpoints of $\lambda$. By Lemma $6.2, \theta$ is arbitrarily small. Then $d\left(p_{1}, p_{2}\right) \approx \theta \cdot s_{D}$. So $\gamma=r^{-1}(\lambda)$ is a circular arc, which is almost a semicircle, of radius approximately equal to $\theta \cdot s_{D} / 2$.

Let $D_{0} \subset \Omega$ be a maximal disk as near as possible to $p_{1}$, such that $\partial D_{0}$ meets both $\partial_{-} \Omega$ and $\partial_{+} \Omega$. Then Dome $(\Omega)$ contains a bending line $\lambda_{0}$, with endpoints $p_{-} \in \partial_{-} \Omega$ and $p_{+} \in \partial_{+} \Omega$. Let $\gamma_{0}=r^{-1}\left(\lambda_{0}\right) \subset \Omega$. Let $s_{0}$ be the radius of $D_{0}$, and let $\alpha$ be the angle between the tangent lines at $p_{-}$and $p_{+}$. Then $s_{D} / s_{0}$ is bounded above (approximately) by 1 , and $\alpha$ is very near to ang $(p)$. See Figure 8.0.

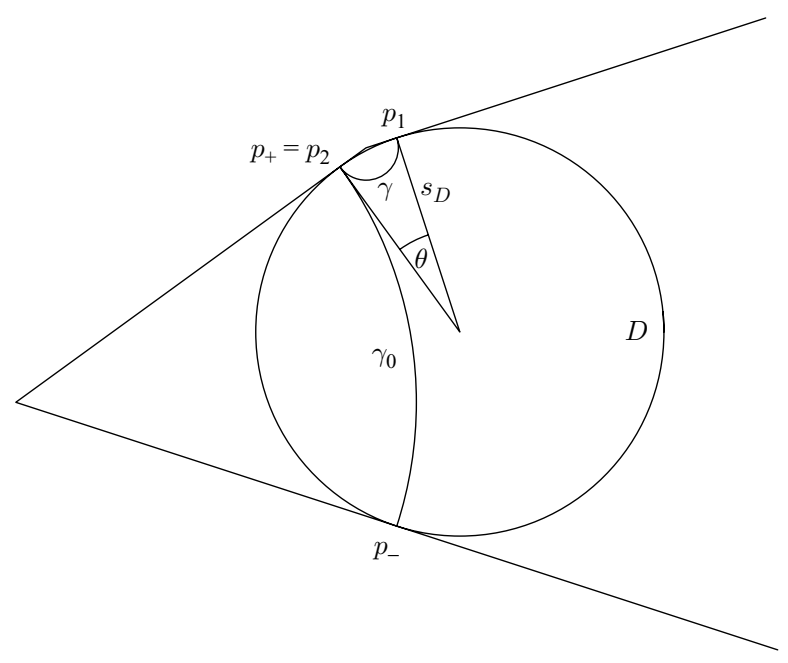

Figure 8.0. This picture illustrates the discussion in $\S 8$. We have taken a case where, among all possible shapes of $\Omega$ and among all possible configurations within $\Omega, D$ happens to meet $\partial_{-} \Omega$ : this makes $s_{D}$ as large as possible consistent with our other assumptions. We have $\gamma=r^{-1}(\lambda)$ and $\gamma_{0}=r^{-1}\left(\lambda_{0}\right)$. The angle at the vertex on the left is approximately equal to ang $(p)$. We illustrate the special situation where $p_{2}$ and $p_{+}$coincide. We also have $D=D_{0}$ and $s_{0}=s_{D}$.

The length of the circular arc $\gamma_{0}$ is $s_{0} \alpha / \tan (\alpha / 2)$ or $2 s_{0}$ if $\alpha=0$. Let $y_{1}$ be the length of the projection of $h(\gamma)$ onto the vertical coordinate of $M$, and let $y_{2}$ be the length of the vertical interval in $M$ through $h\left(p_{1}\right)$. So $y_{1}$ is the angle subtended by $\gamma$ at $p$ (if $\operatorname{ang}(p)>0$ ). The ratio $y_{1} / y_{2}$ is bounded above (approximately) by

$$
\frac{s_{D} \theta / 2}{s_{0} \alpha / \tan (\alpha / 2)} \leqslant \frac{\theta \tan (\alpha / 2)}{2 \alpha} \approx \frac{\theta \tan (\operatorname{ang}(p) / 2)}{2 \operatorname{ang}(p)},
$$

or by $\theta / 4$ if $\operatorname{ang}(p)=0$. (Recall that $\operatorname{ang}(p)<\pi$, so there is no danger that tan might become infinite.) Since $\theta$ tends to zero, this becomes arbitrarily small as we move towards $p$. 
In $M$, as we move towards $+\infty$, the ratio of the euclidean size of fringe bending lines like $\lambda$ in $\S 8$, joining $\partial_{-} \Omega$ ( or $\partial_{+} \Omega$ ) to itself, to the euclidean size of a nearby almost vertical interval joining $\partial_{-} M$ to $\partial_{+} M$, converges to zero. In other words, if we zoom at $p$, the shape looks more and more like a wedge of angle ang $(p)$, and the fringe bending lines become less and less noticeable.

8.1. Definition. We call a bending line like $\lambda$ in $\S 8$, which joins $\partial_{-} \Omega$ (or $\partial_{+} \Omega$ ) to itself, a fringe. We call a bending line like $\lambda_{0}$ in $\S 8$, which joins $\partial_{-} \Omega$ to $\partial_{+} \Omega$ an arch. These definitions are only relevant near $p$. These terms are also used for bending lines of Dome $\left(\Omega_{n}\right)$.

The following lemma is an immediate consequence of the properties of log and of the geometry.

8.2. Lemma. Let $\lambda \subset \operatorname{Dome}(\Omega)$ be an arch. Then $h\left(r^{-1}(\lambda)\right)$ is an analytic curve in $M$. The angles which this curve makes with the vertical converge to zero as we get closer to $p$.

Let $\lambda$ be a bending line of Dome $(\Omega)$. By Lemma 4.8, the endpoints $p_{1}$ and $p_{2}$ of $\lambda$ have unique tangent lines to $\Omega$. There is a unique maximal disk $D \subset \Omega$ with $p_{1}, p_{2} \in \partial D$. Let $P \subset \mathbb{U}^{3}$ be the hyperbolic plane such that $\partial P=\partial D$. We define $P_{\infty}=P \cap$ Dome $(\Omega)$. Then $P_{\infty}$ is a convex polygon in the hyperbolic plane $P$, possibly with an infinite number of sides. We have $\lambda \subset P_{\infty}$.

Now suppose that $p_{1}, p_{2} \in X_{n}$, with tangent lines $\sigma_{1}$ and $\sigma_{2}$ to $\Omega$. Then $D$ is also a maximal disk in $\Omega_{n}$. We define $P_{n}=P \cap \operatorname{Dome}\left(\Omega_{n}\right)$. Now $P_{n}$ is either equal to $\lambda$ or to a finite-sided convex hyperbolic polygon with ideal vertices. In either case, $p_{1}$ and $p_{2}$ are ideal vertices of $P_{n}$ and of $P_{\infty}$. Each ideal vertex of $P_{n}$ is in

$$
\partial D \cap \bar{\Omega} \cap \partial \Omega_{n} \subset \partial D \cap \partial \Omega .
$$

It follows that $P_{n} \subset P_{\infty}$. Either $\lambda=P_{\infty}$ or $\lambda$ is an edge of $\partial P_{\infty}$. We deduce that $\lambda$ is also a bending line of Dome $\left(\Omega_{n}\right)$. This will also hold for all larger values of $n$.

Among the bending lines of $\operatorname{Dome}(\Omega)$ which join $\partial_{-} \Omega$ to $\partial_{+} \Omega$, we therefore see certain bending lines of Dome $\left(\Omega_{n}\right)$, more and more densely as $n$ tends to infinity.

8.3. Definition. In parallel with definition (3.3.a), we define $\psi_{n}: S_{1} \rightarrow S_{u}$ as the composite of homeomorphisms

$$
\psi_{n}: S_{1} \stackrel{g_{n}^{-1}}{\longrightarrow} M_{n} \stackrel{h_{n}^{-1}}{\longrightarrow} \Omega_{n} \stackrel{r_{n}}{\longrightarrow} \text { Dome }\left(\Omega_{n}\right) \stackrel{\iota_{n}}{\longrightarrow} S_{u},
$$

where again $u=\pi \sin (\operatorname{ang}(p) / 2) / \operatorname{ang}(p)$.

Here $g_{n}: M_{n} \rightarrow S_{1}$ is the unique conformal isomorphism sending $h\left(z_{0}\right)$ to 0 and $+\infty$ to $+\infty$. Also $\iota_{n}$ : Dome $\left(\Omega_{n}\right) \rightarrow S_{u}$ is the unique conformal isomorphism sending $x_{0}$ to 0 and $p$ to $+\infty$.

8.4. LEMma. There exist a constant $c>0$ and an $m_{0} \in \mathbb{N}$, both independent of $n(1 \leqslant n<\infty)$, such that the dilatation of $\psi_{n} \mid\left[m_{0}, \infty\right) \times(0, \delta]$ is bounded above by $1+c \delta$. By symmetry of the hypotheses, the same is true for $\psi_{n} \mid\left[m_{0}, \infty\right) \times[1-\delta, 1)$.

Proof. Suppose the first coordinate $t$ of $x=(t, s)$ is large, and that $0<s \leqslant \delta$. In order to prove the claimed bound on the dilatation near $x$, we need only prove the same bound for the dilatation of each nearest point retraction $r_{n}: \Omega_{n} \rightarrow \operatorname{Dome}\left(\Omega_{n}\right)$ 
in a neighbourhood of the point $u=h^{-1}\left(g^{-1}(x)\right) \in \Omega_{n}$. Recall from $\S 7.6$ how $\Omega_{n}$ is constructed.

We now discuss the three possibilities for $u$.

(8.4.1) First, $u$ may lie in a flat of Dome $\left(\Omega_{n}\right)$. In this case the dilatation of $r_{n}$ is 1 .

(8.4.2) Second, $u$ may lie in a conical piece of Dome $\left(\Omega_{n}\right)$, corresponding to a wedge of angle $\alpha$, with $\alpha$ very near to $\operatorname{ang}(p)$. (If $\operatorname{ang}(p)=0$, we interpret 'cone' to include 'cylinder'.) In this case, the bending line is an arch (see Definition 8.1). We apply Lemma 3.1 and see that the dilatation is $1+\cos (\alpha / 2) \sin (\theta)$, where $0<\theta<\pi$ is the angular position of $u$ in its bending line. For the model halfcone or half-cylinder, $\theta$ and $\delta$ can be explicitly computed in terms of each other, using Lemma 4.1 or Remark 4.2. Here our region is not a genuine half-cone or halfcylinder, but only an approximation to such. However, Theorem 7.8 shows that $\delta$ here is a very close approximation to $\delta$ in the model. We deduce that $\theta=O(\delta)$, and so the dilatation is $1+O(\delta)$, as required.

(8.4.3) Third, $u$ may lie in a conical piece of Dome $\left(\Omega_{n}\right)$, corresponding to a wedge of angle $\alpha$, with $\alpha$ very near to $\pi$. In this case, the angle $\theta$ in the formula of Lemma 3.1 bears no relationship with $\delta$, a vertical measurement in $S_{1}$. By taking $m_{0}$ large enough, we can ensure that $\alpha$ is arbitrarily near to $\pi$. Note that $m_{0}$ can be chosen independently of $n$. Then $\cos (\alpha / 2)$ is arbitrarily small, and in particular smaller than $\delta$. So $1+\cos (\alpha / 2) \sin (\theta)<1+\delta$, as required.

\section{The foliation}

The bending lamination and its inverse image under $r$ provide convenient geodesics on which we can compute $r$ precisely. However, we also need to carry out computations in the flats where the bending lamination does not exist. It is therefore helpful to extend the bending lamination to cover some of the flat regions.

We extend the bending lamination of Dome $\left(\Omega_{n}\right)$ to a foliation of those flat pieces $F$ whose boundary components include two bending lines each joining $\partial_{+} \Omega$ to $\partial_{-} \Omega$. We denote these geodesics by $p_{1} q_{1}$ and $p_{2} q_{2}$, where, for $i=1,2, p_{i} \in \partial_{+} \Omega$ and $q_{i} \in \partial_{-} \Omega$. We choose the notation so that $p_{2} q_{2}$ separates $p_{1} q_{1}$ from the boundary point $p \in \partial \Omega$ in $\overline{\text { Dome }\left(\Omega_{n}\right)}$. Let $F \backslash p_{1} q_{2}=F_{1} \cup F_{2}$, where $p_{1} q_{1}$ is a side of $F_{1}$ and $p_{2} q_{2}$ is a side of $F_{2}$. We foliate $F_{1}$ by geodesics ending at $p_{1}$ and $F_{2}$ by geodesics ending at $q_{2}$. The situation is shown in Figure 9.0.

We denote the foliation by $\mathcal{F}_{n}$. Note that this is a foliation of a neighbourhood in Dome $\left(\Omega_{n}\right)$ of the boundary point $p \in \partial$ Dome $\left(\Omega_{n}\right)$, not of the whole of Dome $\left(\Omega_{n}\right)$.

As we move towards $p$, the images in $M_{n}$ under $h_{n}$ of these leaves become more and more nearly vertical.

The following lemma is well known.

9.1. LEmma. Let $\mathcal{F}$ be any foliation of a region $U \subset \mathbb{H}^{2}$ by geodesics, such that the complete geodesics are disjoint in $\mathbb{H}^{2}$. Let $\gamma: \mathbb{R} \rightarrow \mathbb{H}^{2}$ be any geodesic, parametrized by hyperbolic path-length. Let $\theta(t)$ be the angle $\gamma$ makes to $\mathcal{F}$ at $\gamma(t) \in U$. Then $\theta$ is a real-valued lipschitz function of a real variable, with lipschitz constant 1 .

Proof. First note that $\theta^{\prime}(t)=\sin (\theta(t))$ for the foliation of $\mathbb{U}^{2}$ by vertical geodesics. This proves the result for any foliation by geodesics with a single common endpoint in $\partial \mathbb{H}^{2}$. Now let $\beta_{1}$ and $\beta_{2}$ be any two disjoint geodesics. We construct 


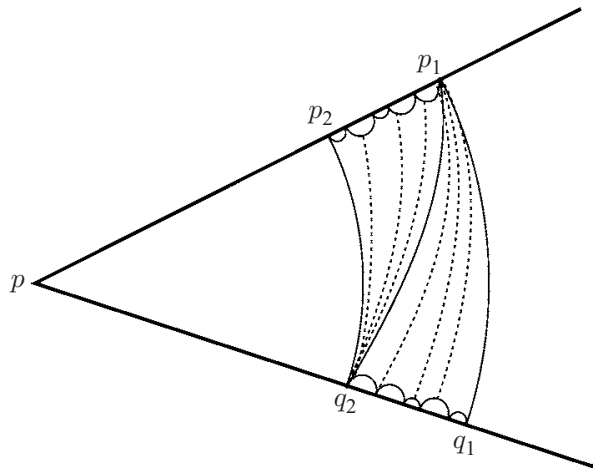

FIgURE 9.0. This illustrates $\S 9$, where the bending lamination is extended to a foliation of a neighbourhood of $p$ by geodesics. The picture should really be drawn on Dome $(\Omega)$, but we have instead drawn the inverse image in $\Omega$ under the nearest point retraction $r$, which is easier to do. Notice the small fringe geodesics. For convenience, we have drawn the boundary of $\Omega$ in two straight pieces, but we must have some deviation from straightness if there is to be any fringe. The solid curves are inverse images of bending lines. The dotted curves are inverse images of the new geodesics introduced in order to create a foliation.

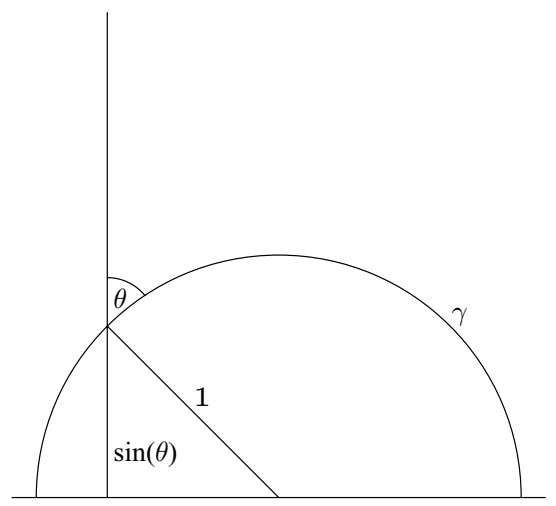

FiguRE 9.1. This diagram illustrates the proof of Lemma 9.1. We show the geodesic $\gamma$ of that proof as a semicircle of radius 1 in the upper half-plane. We work out the relationship between the signed distance $t$ measured along $\gamma$, and the angle that a vertical geodesic makes with $\gamma$. Since the point of interest is at height $\sin (\theta)$, we see that $d t=d \theta / \sin (\theta)$.

a foliation of $\mathbb{H}^{2}$ such that $\beta_{i}$ is a leaf for $i=1,2$. Denote by $\beta_{3}$ one of the two diagonal geodesics joining an endpoint of $\beta_{1}$ to the diagonally opposite endpoint of $\beta_{2}$. Then $\beta_{3}$ separates $\mathbb{H}^{2}$ into two components. The component containing $\beta_{1}$ is foliated by all geodesics through the common endpoint of $\beta_{1}$ and $\beta_{3}$, and the other component is foliated by all geodesics through the common endpoint of $\beta_{2}$ and $\beta_{3}$. The known result for $\theta^{\prime}$ can be applied to each component.

For $i=1,2$, let $\gamma \cap \beta_{i}=\left\{b_{i}\right\}$. By the Mean Value Theorem, the difference in the angle at which $\gamma$ meets $\beta_{1}$ and $\beta_{2}$ is bounded by the hyperbolic distance $d\left(b_{1}, b_{2}\right)$. 
We transfer the foliation $\mathcal{F}_{n}$ on (part of) Dome $\left(\Omega_{n}\right)$, to the various spaces $S_{1}$, $S_{u}, M_{n}$ and $\Omega_{n}$ by using the homeomorphisms

$$
S_{1} \stackrel{g_{n}^{-1}}{\longrightarrow} M_{n} \stackrel{h_{n}^{-1}}{\longrightarrow} \Omega_{n} \stackrel{r_{n}}{\longrightarrow} \text { Dome }\left(\Omega_{n}\right) \stackrel{\iota_{n}}{\longrightarrow} S_{u} .
$$

By abuse of notation, we will denote the corresponding foliations on any of these spaces by $\mathcal{F}_{n}$.

Let $\Gamma_{n}$ be the curve in Dome $\left(\Omega_{n}\right)$ which starts at $x_{0}$, the midpoint of the bending line $\beta_{0}$, and ends at $p$. It follows the locus of highest points on the various leaves of $\mathcal{F}_{n}$, using the height in upper half-space. On a conical piece, $\Gamma_{n}$ follows a geodesic path. We wish to understand how $\Gamma_{n}$ behaves on a flat piece. Using the definition of $\mathcal{F}_{n}$ from $\S 9$, we see that it is sufficient to deal with the case that all the geodesics in $\mathcal{F}_{n}$ lying in this particular flat have a single boundary point in common.

9.2. Lemma. Let $Y_{m, n}=r_{n}\left(h^{-1}\left(g_{n}^{-1}([m, \infty) \times(0,1))\right)\right)$. There is a sequence $\left(\epsilon_{m}\right)_{m \in \mathbb{N}}$ of positive numbers, converging to zero, such that, for each $m$, the total hyperbolic length of $\Gamma_{n}$ within flat pieces meeting $Y_{m, n} \subset$ Dome $\left(\Omega_{n}\right)$ is bounded by $\epsilon_{m}$. In addition, $\Gamma_{n} \cap Y_{m, n}$, parametrized by hyperbolic path-length, is a $\left(1, \epsilon_{m}\right)$ quasigeodesic.

Proof. Let $F$ be a flat piece crossed by $\Gamma_{n}$. By the construction (see Figure 9.0) of $\mathcal{F}_{n}$, we may assume that all the leaves of $\mathcal{F}_{n}$ in $F$ have a boundary point in common. Now $F$ lies in a hyperbolic plane $P$. We choose coordinates so that $\partial P$ is the unit circle and such that the common boundary point of the leaves is $1 \in$ $\partial P$. Suppose that the arch is the geodesic in upper half-space joining $(1,0,0)$ to $(\cos (\theta), \sin (\theta), 0)$. The highest point on the geodesic is

$$
u(\theta)=((1+\cos (\theta)) / 2, \sin (\theta) / 2, \sin (\theta / 2)),
$$

for $0<\theta<2 \pi$. We have

$$
u^{\prime}(\theta)=(-\sin (\theta) / 2, \cos (\theta) / 2, \cos (\theta / 2) / 2) .
$$

The hyperbolic length of $u^{\prime}(\theta)$ is strictly smaller than $2 / \sin (\theta / 2)$. If $m$ is large, then $\theta \approx \pi-\operatorname{ang}(p)$. So $\left\|u^{\prime}(\theta)\right\| \leqslant 2 / \cos (\operatorname{ang}(p) / 2)$.

As $x \in \partial \Omega_{n} \cap \partial \Omega$ moves towards $p \in \partial \Omega_{n} \cap \Omega$ along $\partial \Omega_{n}$, the amount of turning necessary in order to rotate the tangent at $x$ to the (one-sided) tangent at $p$ is independent of $n$. This quantity tends to zero uniformly in $n$ as $x \rightarrow p$. On $\partial \Omega_{n}$, the quantity is the sum of changes in $\theta$ over $\partial F \cap \partial P$. Here the sum is over all flat pieces $F$ through which $\Gamma_{n}$ passes.

To see that $\Gamma_{n}$, parametrized by path-length, is a $\left(1, \epsilon_{m}\right)$-quasigeodesic, let $t_{1}<t_{2}$ be points in the domain of $\Gamma_{n}$, considered as a curve in $Y_{m, n} \subset \operatorname{Dome}\left(\Omega_{n}\right)$. Then $d\left(\Gamma_{n}\left(t_{1}\right), \Gamma_{n}\left(t_{2}\right)\right) \leqslant t_{2}-t_{1}$. Let $\lambda$ be the geodesic from $\Gamma_{n}\left(t_{1}\right)$ to $\Gamma_{n}\left(t_{2}\right)$. Then $\lambda$ has to cross the same conical pieces as $\Gamma_{n}$. The length of $\lambda$ is bounded below by the sum of the lengths of $\lambda$ lying in conical pieces. This is no smaller than the sum of the lengths of $\Gamma_{n}$ in the same conical pieces. In fact, unless $\lambda$ and $\Gamma_{n}$ coincide on such a conical piece, $\lambda$ is definitely longer. This in turn is greater than $t_{2}-t_{1}-\epsilon_{m}$. In symbols, this means

$$
t_{2}-t_{1}-\epsilon_{m} \leqslant d\left(\Gamma_{n}\left(t_{1}\right), \Gamma_{n}\left(t_{2}\right)\right) \leqslant t_{2}-t_{1}
$$

as required. 
The following consequence of Corollary 7.9 helps to show that our intuitive picture of the situation corresponds to reality. Let

$$
u=\pi \sin (\operatorname{ang}(p) / 2) / \operatorname{ang}(p)
$$

and let $\iota_{n}$ : Dome $\left(\Omega_{n}\right) \rightarrow S_{u}$ be the conformal isomorphism taking $x_{0} \in$ Dome $\left(\Omega_{n}\right)$ to $0 \in S_{u}$ and the boundary point $p$ to $+\infty$ (the definition is given above in the simpler situation of Definition 3.3). We recall the definitions of $g_{n}: M_{n} \rightarrow S_{1}$ from $\S 7.7$ and of $\psi_{n}: S_{1} \rightarrow S_{u}$ from Definition 8.3 as the composite

$$
S_{1} \stackrel{g_{n}}{\longrightarrow} M_{n} \stackrel{h}{\longrightarrow} \Omega_{n} \stackrel{r_{n}}{\longrightarrow} \text { Dome }\left(\Omega_{n}\right) \stackrel{\iota_{n}}{\longrightarrow} S_{u} .
$$

In $S_{1}$ and in $S_{u}$, we have the central horizontal geodesic $y=0$. This can be transferred to the other spaces using the homeomorphisms $g_{n}, h, r_{n}$ and $\iota_{n}$. In this way, we get two curves in each of the five spaces.

9.3. Proposition. In each of the five spaces, the two curves are close to each other in the following sense. Firstly, we look at the two curves in $S_{1}$ through a window whose shape is $[m-1, m+1] \times\left(0, \frac{1}{2}\right) \subset S_{1}$. In the other four spaces, the shape is the subset which corresponds under the given homeomorphisms. Secondly, we use Hausdorff metrics with respect to the underlying hyperbolic metric. In $S_{1}$ and $S_{u}$, this is equivalent to using the Hausdorff metric based on the euclidean metric (because the curves lie near the centre of the strip). Thirdly, the tangent directions of nearby points on the two curves are near each other. Fourthly, the errors in how well the two curves approximate each other, both for position and direction, tend to zero as $m$ tends to infinity.

Proof. Proposition 9.3 is proved by comparing in the Hausdorff topology each of the two curves with the corresponding image of $\Gamma_{n}$.

To analyse $\Gamma_{n} \subset \operatorname{Dome}\left(\Omega_{n}\right)$, first examine the conical pieces. The definition shows that $\Gamma_{n}$ runs exactly along the bisector of the wedge of the cone. The result of applying $h \equiv \log$ is a nearly horizontal interval roughly midway between the images of the two edges of the wedge (rough because the centre of the wedge is not exactly the same as the centre for the log-function). Then Theorem 7.8 shows that the image of this piece of $\Gamma_{n}$ in the conical piece is near the midcurve of $S_{1}$. This result extends to the flat pieces by Lemma 9.2, for $\epsilon_{m}$ is small, so the total length inside the flat pieces is small. Moreover, the total geodesic curvature of $\Gamma_{n}$ is small because its turning only takes place within the flat pieces.

Now $\Gamma_{n}$ is also near the midline of $S_{u}$. To see this, note that the midline of $S_{u}$ is a geodesic, while, according to Lemma $9.2, \Gamma_{n}$ is a $\left(1, \epsilon_{m}\right)$-quasigeodesic. These two curves both end at $+\infty$.

In order to see that the statements about directions are true, note that the nearest point retraction $r_{n}$ preserves $\mathcal{F}_{n}$ by definition. The direction orthogonal to $\mathcal{F}_{n}$ is also preserved by $r_{n}$. This is true on flat pieces where $r_{n}$ is conformal, and on conical pieces because it is locally exactly the same as in the model situation. The other maps $g_{n}, h$ and $\iota_{n}$ are conformal, so they have the same property. It follows that a curve which is almost orthogonal to $\mathcal{F}_{n}$ in one of the five spaces has that same property when transferred to the other spaces.

9.4. Example. It is tempting to think that a central line in $M_{n}$ could be added to Proposition 9.3 so as to give three asymptotic curves, rather than two. This can 
indeed be done if $\operatorname{ang}(p)>0$. But if $p=\infty$ and $\operatorname{ang}(p)=0$, there are counterexamples. For example, consider the subset of $\mathbb{R}^{2}$ lying between the positive $x$-axis and the upper half of the parabola $y^{2}=x$. This is a convex region $\Omega$, equal to $M$, and there is no central line corresponding to $p=\infty$. A hyperbolic geodesic in $\Omega$ limiting at $\infty$ cannot be asymptotic to a straight line.

\subsection{The leaves of $\mathcal{F}_{n}$ near $p$}

We now discuss the shape of a leaf $\lambda$ of $\mathcal{F}_{n}$ near the boundary point of interest $(p$ or $+\infty)$. We start in $\Omega_{n}$, normalizing coordinates so that $\lambda$ has length 1 . If $\operatorname{ang}(p)>0$ and $p$ is finite, then a leaf $\lambda$ of $\mathcal{F}_{n}$ in $\Omega_{n}$ is necessarily a circular arc, provided $\lambda$ is near enough to $p$. As $\lambda$ moves towards $p$, we continually rescale, normalizing with $\lambda$ of length 1 , the centre of the circle containing the arc at zero, and the midpoint of $\lambda$ real and positive.

As we do this, $p$ tends towards the centre of the normalized circle containing $\lambda$ as a subarc of length 1 . If $\operatorname{ang}(p)>0$ and $p=\infty$, we proceed in the same way, but now the centre of the circle has no particular relationship to $p$. In each case, the radius of the circle whose circumference has $\lambda$ as a subarc converges to $1 / \operatorname{ang}(p)$. If $\operatorname{ang}(p)=0$, then $\lambda$ converges to an interval of length 1 and, after normalization, $p$ tends to infinity. In more detail, the tangent lines to $\partial \Omega_{n}$ are orthogonal to the leaves of $\mathcal{F}_{n}$. They converge to the limit tangent lines at $p$. So, after rescaling, their point of intersection is ever closer to $p$.

It follows that, on $M_{n}$ near the boundary point $+\infty$, the leaves of $\mathcal{F}_{n}$ are nearly vertical curves (at any rate, away from the boundary curves), the tangent directions tending to the vertical as $m$ tends to infinity, uniformly in $n$ and in the position along the leaf. By Theorem 7.8, it follows that given a small $\delta>0$, on $[m, \infty) \times$ $\left[-\frac{1}{2}+\delta / 2, \frac{1}{2}-\delta / 2\right] \subset S_{1}, \mathcal{F}_{n}$ is nearly vertical, the error converging to zero as $m$ tends to infinity, uniformly in $n$.

We see from Lemma 9.2 that the isometry $\iota_{n}$ sends the hyperbolic quasigeodesic $\Gamma_{n}$ to near the central line of $S_{u}$. On compact subsets of $S_{u}$, tangent vectors to $\mathcal{F}_{n}$ therefore converge to the vertical.

Recall from $\S 8$ that fringe effects arise as follows: two tangent lines to a large maximal disk $D$ touching both sides of $\partial \Omega_{n}$, tangency points near each other, relative angle nearly $\pi$ to each other. If $\partial D$ is normalised so that the leaf $\lambda$ has length 1 , its radius settles down to approximately $\tan (\alpha / 2) / \alpha$ (compare with the wedge case). Fringe effects arise from small arcs of nearly semicircles, orthogonal to both the tangent lines, each with an end point at a point of tangency. Their euclidean radius (after normalisation) is small (on the order of $\epsilon_{m}$ which is the angle between the renormalised tangent lines). The image of the fringe under $h_{n}$ has small height in $M_{n}$. It follows using Theorem 7.8 that the image of the fringe has small height in $S_{1}$.

Consequently $\delta \rightarrow 0$ as $m \rightarrow \infty$. Therefore the fringe effects occur within distance $\delta / 4$ of $\partial S_{1}$, for large enough $m$. The choice $\delta / 4$ prevents bumping into the $\delta / 2$-line used above.

\section{A key proposition}

Now we come to one of the main intermediate results required in the proof of Theorem 7.1. 
10.1. Proposition. Let $u=\pi \sin (\operatorname{ang}(p) / 2) / \operatorname{ang}(p)$ and let $\tau_{z}: \mathbb{C} \rightarrow \mathbb{C}$ be translation by $z$. For all $m, n \in \mathbb{N}$, consider the composite

$$
S_{1} \stackrel{\tau_{m}}{\longrightarrow} S_{1} \stackrel{\psi_{n}}{\longrightarrow} S_{u} \stackrel{\tau_{-m^{\prime}}}{\longrightarrow} S_{u}
$$

where $m^{\prime} \in \mathbb{R}$ is chosen so that the composite sends 0 to a point on the $y$-axis. As $m$ tends to infinity, the composite converges on compact subsets of $S_{1}$ to the model map $\Psi$, that is, to the map $(t, s) \mapsto\left(t,-u / 2+f_{\alpha}\left(s+\frac{1}{2}\right)\right)$. (See Definition 3.3 and equation (3.3.b).) The convergence is uniform on the compact subset and with respect to $n$. (See the statement of Theorem 7.8 for the meaning of uniform convergence with respect to $n$.)

Proof. The proof is based on rescaling. It seems clearest to separate the argument for different cases, even though the proofs of the different cases run along similar lines.

We start with $p$ finite and $0<\operatorname{ang}(p)<\pi$. We take $p=0$. The two tangents at $p$ are the rays $\arg (z)=-\operatorname{ang}(p) / 2$ and $\arg (z)=\operatorname{ang}(p) / 2$. Let $z_{n, m}=$ $h^{-1}\left(g_{n}^{-1}(m)\right)=\exp \left(-g_{n}^{-1}(m)\right) \in \Omega_{n}$. For $m$ large, $\left|z_{n, m}\right|$ is very small. Let $\Omega_{n, m}$ be the result of scaling $\Omega_{n}$ by a factor $\left|z_{n, m}\right|^{-1}$. In $\Omega_{n, m}$, our point of interest $z_{n, m}$ is sent into the unit circle.

The composite in the statement of Proposition 10.1 is factored into two other composites. Each of these is uniformly convergent, and we will be able to compute the limits. The first composite is

and the second is

$$
S_{1} \stackrel{\tau_{m}}{\longrightarrow} S_{1} \stackrel{g_{n}^{-1}}{\longrightarrow} M_{n} \stackrel{h^{-1}}{\longrightarrow} \Omega_{n} \stackrel{1 /\left|z_{n, m}\right|}{\longrightarrow} \Omega_{n, m}
$$

$$
\Omega_{n, m} \stackrel{r_{n, m}}{\longrightarrow} \text { Dome }\left(\Omega_{n, m}\right) \stackrel{\left|z_{n, m}\right|}{\longrightarrow} \text { Dome }\left(\Omega_{n}\right) \stackrel{\iota_{n}}{\longrightarrow} S_{u} \stackrel{\tau_{-m^{\prime}}}{\longrightarrow} S_{u} .
$$

Here the map between domes is the hyperbolic isometry of $\mathbb{H}^{3}$ which is equal to multiplication by the real number $\left|z_{n, m}\right|$ on $\partial \mathbb{H}^{3}=\mathbb{C}$.

We claim that the sequence of first composites converges to the model map $S_{1} \rightarrow U_{\operatorname{ang}(p)}$ (using the notation of Lemma 3.1), given by $z \mapsto \exp (-\operatorname{ang}(p) z)$. To see this, note first that from Corollary 7.9, Theorem 7.8 and the fact that $h^{-1}(z)=\exp (-z)$, we can deduce that the image of $y=0$ in $S_{1}$ is a curve in $\Omega_{n}$ which ends at $p$ and is asymptotic to the bisector of the angle between the two tangents at $p$. It follows that $z_{n, m} /\left|z_{n, m}\right|$ converges to 1 as $m$ tends to infinity, and the convergence is uniform with respect to changes in $n$. Furthermore, the euclidean distance of $z_{n, m} /\left|z_{n, m}\right|$ to $\partial \Omega_{n, m}$ converges to $\sin (\operatorname{ang}(p) / 2)$. The Koebe One Quarter Theorem now implies that the sequence of derivatives of the first composite at 0 is uniformly bounded away from 0 and $\infty$.

The second composite has the form demanded by Theorem 5.3, namely it is a nearest point retraction, followed by an isometry Dome $\left(\Omega_{n, m}\right) \rightarrow S_{u}$. In order to apply Theorem 5.3, we also need to check normalizations. By construction, $z_{n, m} /\left|z_{n, m}\right|$ is sent to a point in $S_{u}$ on the $y$-axis. By Proposition 9.3 , this point is near $y=0$. It therefore converges to $0 \in S_{u}$ as $m$ tends to infinity. Consider the geodesic in $\Omega_{n, m}$ from $z_{n, m} /\left|z_{n, m}\right|$ to 0 . By Proposition 9.3 , this geodesic is sent to a curve in $S_{u}$ which is asymptotic to $y=0$. Theorem 5.3 now tells us that the limit is the map

$$
U_{\text {ang }(p)} \stackrel{r}{\longrightarrow} \text { Dome }\left(U_{\text {ang }(p)}\right) \stackrel{\iota}{\longrightarrow} S_{u}
$$


where $\iota$ is the hyperbolic isometry which

(i) preserves orientation,

(ii) sends $r(1)$ to 0 and

(iii) sends the geodesic along the top of the cone to the central horizontal line of $S_{u}$.

Since the uniform limit of a composition is the composition of the uniform limit, Proposition 10.1 now follows if $p$ is finite.

When $p=\infty$ and $\operatorname{ang}(p)>0$, the same proof works, though now $z_{n, m}$ tends to infinity.

When $p=\infty$ and $\operatorname{ang}(p)=0$, the proof is very slightly different. We define $z_{n, m}=$ $x_{n, m}+i y_{n, m}=g_{n}^{-1}(m)$. Then $x_{n, m}$ tends to $+\infty$ as $m$ tends to infinity, uniformly with respect to $n$. Also $y_{n, m} / x_{n, m}$ tends to zero. Let $\sigma_{n, m}(z)=a(m, n) z+z_{n, m}$, where $a(m, n)$ is the length of the vertical interval from $\partial_{-} M$ to $\partial_{+} M$ with $x$ coordinate equal to $m$. (See $\S 7.7$.) We define $\Omega_{n, m}=\sigma_{n, m}^{-1}\left(\Omega_{n}\right)$. As in the case when $p=0$ and $\operatorname{ang}(p)>0$, we factorize the map $S_{1} \rightarrow S_{u}$ in the statement of Proposition 10.1 as

$$
S_{1} \stackrel{\tau_{m}}{\longrightarrow} S_{1} \stackrel{g_{n}^{-1}}{\longrightarrow} \Omega_{n} \stackrel{\sigma_{n, m}^{-1}}{\longrightarrow} \Omega_{n, m}
$$

with

$$
\Omega_{n, m} \stackrel{r}{\longrightarrow} \text { Dome }\left(\Omega_{n, m}\right) \stackrel{\sigma_{n, m}}{\longrightarrow} \text { Dome }\left(\Omega_{n}\right) \stackrel{\iota_{n}}{\longrightarrow} S_{u} \stackrel{\tau_{-m^{\prime}}}{\longrightarrow} S_{u} .
$$

The first composite sends 0 to 0 . It sends the horizontal line $y=0$ to a curve in $\Omega_{n, m}$ which, near $0 \in \Omega_{n, m}$, is nearly horizontal. It follows that the limit is the identity map. The second composite sends $0 \in \Omega_{n, m}$ to a point on the $y$-axis. By Proposition 9.3, this point converges to $0 \in S_{u}$. Also the second composite sends a horizontal interval of length 2 and centre 0 in $\Omega_{n, m}$ to a nearly horizontal curve passing near $0 \in S_{u}$. From Theorem 5.3, this converges to the model map.

This proves Proposition 10.1 when $p=\infty$ and $\operatorname{ang}(p)=0$.

\subsection{The derivative of $\psi_{n}$}

The next thing we have to do is to estimate the derivative of the map $\psi_{n}$ : $S_{1} \rightarrow S_{u}$ (see Definition 8.3). Since the derivative of translation is the identity, this is the same as estimating the derivative of the composite of Proposition 10.1. In particular, we want to estimate, for small $\delta>0$, the derivative of $\psi_{n} \mid[m-1$, $m+1] \times\left\{\frac{1}{2}-\delta\right\}$. The estimate for the $\psi_{n} \mid[m-1, m+1] \times\left\{-\frac{1}{2}+\delta\right\}$ will be the same, by symmetry of the hypotheses. For this discussion, we will use, in an essential way, the approximations $\Omega_{n}$ to $\Omega$. (Previous uses of the approximation were not essential.)

The nearest point retraction $r_{n}: \Omega_{n} \rightarrow$ Dome $\left(\Omega_{n}\right)$ is real analytic in the interior of each conical, cylindrical or flat (that is, hemispherical) piece. On a bending line marking the transition between such pieces, $r_{n}$ is not differentiable. The restriction of $r_{n}$ to any leaf of $\mathcal{F}_{n}$ is real analytic.

10.3. Proposition. There is an $m_{0}$ such that the restriction of $\psi_{n}$ to the closed interval $\ell=[m-1, m+1] \times\left\{\frac{1}{2}-\delta\right\}$ has the following properties.

(10.3.1) The tangent vector to the piecewise analytic curve $\psi_{n}(\ell)$ (which has two possible values at the endpoint of an analytic piece) is nearly horizontal. The 
convergence to the horizontal, as $m$ tends to infinity, is uniform with respect to $n$. (The positive integer $n$ can vary as $m$ varies.)

(10.3.2) In (10.3.1), the length of the tangent vector is $1+O(\delta)$. The constants in the $O$-notation are independent of $n$ and of $m \geqslant m_{0}$.

Proof. By $\S 9.5$, the leaves of $\mathcal{F}_{n}$ in $S_{1}$ and $S_{u}$ are nearly vertical if $m$ is large. The factors $g_{n}, h$ and $\iota_{n}$ preserve angles and the foliation $\mathcal{F}_{n}$. The nearest point retraction $r_{n}$ does not preserve angles. But it does preserve the foliation $\mathcal{F}_{n}$ and the direction orthogonal to the leaves of this foliation. Since $r_{n}$ has dilatation at most 2 (by Theorem 5.1), very small angles which are almost horizontal or almost vertical in $S_{1}$ are sent by $\psi_{n}$ to angles which are small and almost horizontal or almost vertical respectively. In particular, the curve $\psi_{n}\left([m-1, m+1] \times\left\{\frac{1}{2}-\delta\right\}\right)$ is almost horizontal in $S_{u}$. This proves (10.3.1).

To prove (10.3.2), we examine further the composite $\tau_{-m^{\prime}} \circ \psi_{n} \circ \tau_{m}: S_{1} \rightarrow S_{u}$ introduced in Proposition 10.1. We describe the effect of the composite on a leaf of $\mathcal{F}_{n}$ which passes through some fixed compact subset $C \subset S_{1}$. We parametrize the leaf in $S_{1}$ by euclidean path-length, and we examine the effect of composing the parametrization with the above composite.

Since we are assuming $m$ large, the leaf is almost vertical in $C$. In each of the spaces we look at, the leaf will follow, more and more closely on a compact subset, the model direction. We will leave details of this to the reader, and concentrate on explaining more subtle features.

We compose the path-length parametrization of a leaf in $S_{1}$ with the map

$$
S_{1} \stackrel{\tau_{m}}{\longrightarrow} S_{1} \stackrel{g_{n}^{-1}}{\longrightarrow} M_{n} .
$$

By Theorem 7.8, on the compact subset $C \subset S_{1}$, the parametrization of the image curve in $M_{n}$ is $C^{1}$-near to some parametrization proportional to path-length.

The next map to apply is $h^{-1}: M_{n} \rightarrow \Omega_{n}$ which is $z \mapsto \exp ( \pm z)$ or the identity. The image in $\Omega_{n}$ of our leaf is an arc of a circle whose centre is arbitrarily near 0 if $\operatorname{ang}(p)>0$, and is an interval or arc of a circle with very large radius if $\operatorname{ang}(p)=0$. In the case of an arc of a circle, the parametrization is $C^{1}$-near to a parametrization proportional to the angle subtended at the centre. For both arcs of circles and intervals in $\Omega_{n}$, the parametrization is $C^{1}$-near a parametrization proportional to path-length. The constant of proportionality is the path-length of the arc or interval.

If we stick to a single leaf of $\mathcal{F}_{n}$ in $\Omega_{n}$, the effect of the nearest point retraction can be computed exactly, using the map $f_{\alpha}$ (see equation (3.3.b)). Let $P$ be the hyperbolic plane tangent to Dome $\left(\Omega_{n}\right)$ and containing the corresponding leaf in Dome $\left(\Omega_{n}\right)$. Then the subscript $\alpha$ of $f_{\alpha}$ is the angle between the two tangents to $P$ at the two ends of the leaf. So $\alpha$ converges to $\operatorname{ang}(p)$ as $m$ tends to infinity, and $f_{\alpha}$ is $C_{\infty}$-approximated by $f_{\text {ang }(p)}$. The derivative of $\psi_{n}$ along a leaf of $\mathcal{F}_{n}$ is therefore approximately $f_{\operatorname{ang}(p)}^{\prime}(\delta)=1+O(\delta)$ at a point of the curve $\psi_{n}([m-1, m+1] \times$ $\left.\left\{-\frac{1}{2}+\delta\right\}\right)$.

If we are in a flat piece of Dome $\left(\Omega_{n}\right)$, the nearest point retraction is conformal, and the directional derivative has the same magnitude in each direction. In particular, the length of the derivative vector with respect of $\tau_{-m^{\prime}} \circ \psi_{n} \circ \tau_{m}$ along $\tau_{-m^{\prime}} \psi_{n} \tau_{m} \mid[-1,+1] \times\left\{\frac{1}{2}-\delta\right\}$ with respect to the parameter $t \in[-1,1]$ is $1+O(\delta)$.

If we are in a conical piece, we can argue directly from the shape to prove that the derivative has magnitude arbitrarily near 1 . However, we prefer to follow the style of 
reasoning just used, which gives a weaker result. Namely, Lemma 8.4 shows that the nearest point retraction is $(1+O(\delta))$-quasiconformal along the curve of interest. It follows that the directional derivative in any direction must have magnitude $1+O(\delta)$. This completes the proof of (10.3.2).

\subsection{A quasiconformal map $S_{1} \rightarrow S_{u}$}

Using the previous results, we now define a quasiconformal map $\rho_{n, \delta}: S_{1} \rightarrow S_{u}$ which will give rise to the sequence we require for the proof of Theorem 7.1. The definition depends on a certain positive integer $L_{0}$. We will prove that $L_{0}$ can be chosen large enough so that all the properties required of $\rho_{n, \delta}$ are true. We choose the left-most leaf $\lambda$ of $\mathcal{F}_{n}$ which passes through the strip $\mathbb{R} \times\left[-\frac{1}{2}+\delta, \frac{1}{2}-\delta\right]$ so that its intersection with the strip is entirely (weakly) to the right of the vertical line $x=L_{0}$.

(10.4.1) If $s \leqslant-\frac{1}{2}+\delta$ or $s \geqslant \frac{1}{2}-\delta$, then $\rho_{n, \delta}(t, s)=\psi_{n}(t, s)$.

(10.4.2) If $-\frac{1}{2}+\delta \leqslant s \leqslant \frac{1}{2}-\delta$ and $(t, s)$ is to the left of $\lambda$, then $\rho_{n, \delta}(t, s)=\psi_{n}(t, s)$.

(10.4.3) Let $t \geqslant L_{0}+1$. On the vertical interval $\{t\} \times\left[-\frac{1}{2}+\delta, \frac{1}{2}-\delta\right] \subset S_{1}, \rho_{n, \delta}(t, s)$ is a linear interpolation of the values already defined at the two endpoints.

(10.4.4) We have not yet defined $\rho_{n, \delta}$ on an almost rectangular region whose four sides are given by $\lambda, y=\frac{1}{2}-\delta, y=-\frac{1}{2}+\delta$, and $x=L_{0}+1$. Using Lemma 10.5, we will define $\rho_{n, \delta}$ so that it is $K$-quasiconformal, for some fixed $K$, independent of $n$, $\delta$ and $L_{0}$. Here $\delta>0$ must be chosen small enough and $L_{0}$ large enough, so that the basic topological features of the picture of the map $\rho_{n, \delta}$ are as we expect them to be. For example, $L_{0}$ must be chosen greater than the $m_{0}$ of Proposition 10.3, and $\delta$ must be chosen small enough so that the image of the (almost) rectangular gap really is an (almost) rectangle.

We start by filling the gap at (10.4.4). Recall that the cross-ratio of a quadruple of points in $\mathbb{C P}^{1}$ is a point in $\mathbb{C P}^{1}$, defined provided that at least three of the four points are distinct. We fix conventions by defining the cross-ratio $X(z, 1 ; 0, \infty)=z$. Given a Jordan region, $U \subset \mathbb{S}^{2}$ and points $x_{1}, x_{2}, x_{3}, x_{4} \in \partial U$, at least three of which are distinct, we define the cross-ratio $X_{U}\left(x_{1}, x_{2} ; x_{3}, x_{4}\right)$ by choosing a Riemann mapping $f: U \rightarrow \mathbb{H}^{2}$, extending $f$ continuously to $f: \bar{U} \rightarrow \overline{\mathbb{H}^{2}}$, and then taking the usual cross-ratio $X\left(f x_{1}, f x_{2} ; f x_{3}, f x_{4}\right)$. The result is independent of the choice of Riemann mapping.

Given two Jordan regions $U$ and $V$, and a homeomorphism $\rho: \partial U \rightarrow \partial V, \rho$ is said to be quasisymmetric if it does not distort cross-ratios too much. More precisely, the standard theory (due to Ahlfors), says that we can fix a basepoint $x_{0} \in \partial U$ and confine our attention to counter-clockwise quadruples $\left(a, b, c, x_{0}\right)$ with $X_{U}\left(a, c ; b, x_{0}\right)=-1$. Here $a, b, c$ and $x_{0}$ are distinct points in $\partial U$. We say that $\rho$ is $k$-quasisymmetric if the image cross-ratio satisfies $1 / k \leqslant X_{V}(\rho(a), \rho(c)$; $\left.\rho(b), \rho\left(x_{0}\right)\right) \leqslant k$ for all choices of $a, b, c$ in $\partial U$ giving cross-ratio -1 as already explained.

In order to fill in the gap at (10.4.4), we apply these considerations to $\rho_{n, \delta}$. The basepoint for computation of cross-ratios will be the upper right-hand corner of the rectangular region, explicitly $\left(L_{0}+1, \frac{1}{2}-\delta\right) \in S_{1}$.

10.5. Lemma. There exist $k \geqslant 1, \delta_{0}>0$ and $m_{0} \in \mathbb{Z}$, such that, for all $n$, for all $0<\delta \leqslant \delta_{0}$ and for all $L_{0} \geqslant m_{0}$, the restriction of $\rho_{n, \delta}$ to the boundary of the rectangular gap is $k$-quasisymmetric. 
Proof. Suppose this is false. Then we can construct the following data.

(10.5.1) A sequence $\left(\delta_{i}\right)_{i \in \mathbb{N}}$ tending monotonically to zero.

(10.5.2) A sequence $\left(L_{i}\right)_{i \in \mathbb{N}}$ tending monotonically to infinity.

(10.5.3) A sequence $\left(n_{i}\right)_{i \in \mathbb{N}}$ which does not necessarily converge.

(10.5.4) The corresponding maps

$$
T_{i}=\tau_{-m^{\prime}} \circ \rho_{n, \delta} \circ \tau_{m}: S_{1} \rightarrow S_{u},
$$

with $m=L_{i}$, defined except on the interiors of the rectangular gaps.

(10.5.5) For each $i$, an almost rectangular domain gap $U=U_{i}$ and an almost rectangular image gap $V=V_{i}$, corresponding to $n=n_{i}, \delta=\delta_{i}$ and $L_{0}=L_{i}$.

(10.5.6) Convergent sequences $\left(a_{i}\right)_{i \in \mathbb{N}},\left(b_{i}\right)_{i \in \mathbb{N}}$ and $\left(c_{i}\right)_{i \in \mathbb{N}}$, with $a_{i}, b_{i}, c_{i} \in \partial U_{i}$, with limits $a_{\infty}, b_{\infty}$ and $c_{\infty}$ respectively, and cross-ratio $X_{U}\left(a_{i}, c_{i} ; b_{i}, x_{0}\right)=-1$.

The data has the following properties.

(10.5.7) The cross-ratio $X_{V}\left(T_{i}\left(a_{i}\right), T_{i}\left(c_{i}\right) ; T_{i}\left(b_{i}\right), T_{i}\left(x_{0}\right)\right)$ converges either to infinity or to zero.

(10.5.8) The sequence $\left(U_{i}\right)_{i \in \mathbb{N}}$ converges to the rectangle $U_{\infty}=[0,1] \times\left[-\frac{1}{2}, \frac{1}{2}\right]$.

(10.5.9) The sequence $\left(V_{i}\right)_{i \in \mathbb{N}}$ converges to the rectangle

$$
V_{\infty}=[0,1] \times[-u / 2, u / 2] .
$$

A theorem of Rado (see [6, Theorem 2.11]) shows that the Riemann maps for the regions $U_{i}$ (normalized for example by sending three of the four corners to three fixed points in the boundary of the disk) converge to the Riemann map for the rectangle $U_{\infty}$, and the Riemann maps for the regions $V_{i}$ converge to the Riemann map for the rectangle $V_{\infty}$. Rado's theorem uses the fact that the boundaries of the regions are parametrized, and the parametrizations converge uniformly to the parametrization of the boundary of the limit.

Note that the boundary maps $T_{i}: \partial U_{i} \rightarrow \partial V_{i}$ together with their inverses $T(i)^{-1}$ : $\partial V_{i} \rightarrow \partial U_{i}$ are piecewise differentiable maps, with uniformly bounded derivatives if we parametrize by path-length. We have four euclidean distances in $S_{1}$, namely $\left|x_{0}-a_{i}\right|,\left|a_{i}-b_{i}\right|,\left|b_{i}-c_{i}\right|$ and $\left|c_{i}-x_{0}\right|$. If the smallest of the four distances is bounded below away from zero, then the uniform boundedness of derivatives shows that the same is true for the images under $T_{i}$. But this is impossible, because then the image cross-ratio would tend neither to zero nor to infinity. We therefore assume that, independently of $i$, the distance occupying the same position (1,2,3 or 4) is the smallest of the four, and converges to zero.

Let $\left(v_{i}, w_{i}\right)$ denote that pair in the four-element set

$$
\left\{\left(x_{0}, a_{i}\right),\left(a_{i}, b_{i}\right),\left(b_{i}, c_{i}\right),\left(c_{i}, x_{0}\right)\right\}
$$

which has smallest distance apart. We define a similarity $\sigma_{U, i}$ with multiplicative factor $1 /\left|v_{i}-w_{i}\right|$, such that $\sigma_{U, i}\left(v_{i}\right)$ converges to some point in $\mathbb{C}$. Now look at the image of each of the four 'corners' of $U_{i}$ under $\sigma_{U, i}$. We may assume that each of these images converges, either to a finite point, or to infinity. At most one of the four corners has a finite limit, because the distance between two corners, after applying $\sigma_{U, i}$ is very large. If this happens, choose the constant term in the similarity $\sigma_{U, i}$ so that it maps that corner to 0 . If no corner gives a finite limit, choose $\sigma_{U, i}$ so that $\sigma_{U, i}\left(v_{i}\right)=0$ for each $i$.

We define the similarity $\sigma_{V, i}$ with multiplicative constant $1 /\left|v_{i}-w_{i}\right|$, and so that, for each $i, \hat{T}_{i}=\sigma_{V, i} \circ T_{i} \circ \sigma_{U, i}^{-1}$ sends 0 to 0 . We set $\hat{U}_{i}=\sigma_{U, i}\left(U_{i}\right)$ and $\hat{V}_{i}=\sigma_{V, i}\left(V_{i}\right)$. So $\hat{U}_{i}$ and $\hat{V}_{i}$ are very large almost rectangles. 
If one of the corners of $\hat{U}_{i}$ and $\hat{V}_{i}$ is at 0 for each $i$, then $\hat{U}_{i}$ and $\hat{V}_{i}$ converge to one of the coordinate quadrants of $\mathbb{C}$. Otherwise, $\hat{U}_{i}$ and $\hat{V}_{i}$ converge to half-planes (with either vertical or horizontal boundary). Riemann maps with image $\mathbb{H}^{2}$ for these limits $\hat{U}_{\infty}$ and $\hat{V}_{\infty}$ are given by rotation about 0 through a multiple of $\pi / 2$, possibly followed by squaring $z \mapsto z^{2}$.

The derivatives of $\hat{T}_{i}$ are the same as those of $T_{i}$. From Proposition 10.3, we see that the limit $\hat{T}_{\infty}$ of the $\hat{T}_{i}$ is the identity on horizontal boundaries. On a lefthand vertical boundary it is equal to multiplication by $f_{\text {ang }(p)}^{\prime}(s)$, where $s \in[0,1]$ corresponds to the limit $v_{\infty}$ of $\left(v_{i}\right)_{i \in \mathbb{N}}$. On a right-hand vertical boundary, $\hat{T}_{\infty}$ is multiplication by $u=\pi \sin (\operatorname{ang}(p) / 2) / \operatorname{ang}(p)$.

Now $\hat{v}_{\infty}$ and $\hat{w}_{\infty}$ are finite, by construction, corresponding to two of the four sequences $\left(\sigma_{U, i}\left(a_{i}\right)\right)_{i \in \mathbb{N}},\left(\sigma_{U, i}\left(b_{i}\right)\right)_{i \in \mathbb{N}},\left(\sigma_{U, i}\left(c_{i}\right)\right)_{i \in \mathbb{N}},\left(\sigma_{U, i}\left(x_{0}\right)\right)_{i \in \mathbb{N}}$. Recall that we have chosen $\left|u_{i}-v_{i}\right|$ to be the smallest of four choices. Therefore the other two limits are distinct from $\hat{v}_{\infty}$ and $\hat{w}_{\infty}$, so that three of the four limit points are distinct and their cross-ratio is well defined. By definition, the cross-ratio $X_{U_{i}}\left(a_{i}, c_{i} ; b_{i}, x_{0}\right)=-1$. By Rado's theorem, cited above, the cross-ratio of the limits $X_{U_{\infty}}\left(\hat{a}_{\infty}, \hat{c}_{\infty} ; \hat{b}_{\infty}, \hat{x}_{\infty}\right)=-1$. It follows that the four limits are distinct points of the boundary of the quadrant or half-plane $\hat{U}_{\infty}$. It further follows that the four image points in $\partial \hat{V}_{\infty}$ are distinct. So their cross-ratio is neither 0 nor $\infty$. But this is a contradiction, and so the proof of Lemma 10.5 is complete.

\section{Completion of Theorem 7.1}

Proof of Theorem 7.1. By Lemma 10.5, we can fill the gap in the definition of $\rho_{n, \delta}$ with a $k_{1}$ quasiconformal homeomorphism, where $k_{1}>1$ is a uniform constant. (The method of proof of Lemma 10.5 could be used to give an explicit value of $k$ in Lemma 10.5, and therefore of $k_{1}$, in terms of $\operatorname{ang}(p)$.)

We first prove that $\rho_{n, \delta}: S_{1} \rightarrow S_{u}$ is a homeomorphism (for small $\delta$ and large $\left.m_{0}\right)$. This follows because it is injective and sends the boundary to the boundary. Therefore, by topology, it is surjective and a homeomorphism.

In order to show that it is quasiconformal, with an appropriate constant, we need to compute the derivative on $\left(m_{0}+1, \infty\right) \times\left[-\frac{1}{2}+\delta, \frac{1}{2}-\delta\right]$. For an appropriate choice of $\lambda(s)$, the $2 \times 2$ derivative matrix at $(x, s)$ is equal to

$$
\left[\lambda(s) \frac{\partial \psi_{n}}{\partial x}\left(p_{1}\right)+(1-\lambda(s)) \frac{\partial \psi_{n}}{\partial x}\left(p_{2}\right) \frac{\psi_{n}\left(x, \frac{1}{2}-\delta\right)-\psi_{n}\left(x,-\frac{1}{2}+\delta\right)}{1-2 \delta}\right],
$$

where $p_{1}=\left(x, \frac{1}{2}-\delta\right)$ and $p_{2}=\left(x, \frac{1}{2}+\delta\right)$, and each entry is a column vector of length 2. For large enough $m_{0}$, this matrix can be estimated as

$$
\left[\begin{array}{cc}
1+O(\delta) & O(\delta) \\
O(\delta) & u+O(\delta)
\end{array}\right]
$$

where the constants in the $O(\delta)$ terms do not depend on $n$. By taking $\delta$ sufficiently small, we can make the dilatation arbitrarily near $u$.

Now $\rho_{n, \delta}: S_{1} \rightarrow S_{u}$ is a sequence of quasiconformal homeomorphisms with a uniform quasiconformal constant $K$. These all send 0 to 0 and $+\infty$ to $+\infty$, and so there is a limit $\rho_{\delta}: S_{1} \rightarrow S_{u}$ with quasiconformal constant not exceeding $K$. The $\rho_{n, \delta}$ correspond to quasiconformal homeomorphisms $\Omega_{n} \rightarrow$ Dome $\left(\Omega_{n}\right)$ which extend continuously to the identity map on $\partial \Omega_{n}$. By Theorem $5.2, \rho_{\delta}$ corresponds 
to a quasiconformal homeomorphism $\Omega \rightarrow$ Dome $(\Omega)$ which extends continuously to the identity on $\partial \Omega$. If we let $\delta$ tend to zero, we obtain the type of sequence necessary in order to find the substantial boundary dilatation.

At first sight the proof only gives an upper bound. But suppose the value of the substantial dilatation were lower than the known value for a true wedge. Repetition of the rescaling process would result in the contradictory finding that the value for the wedge is less than the known exact value.

This completes the proof of Theorem 7.1.

\section{References}

1. D. B. A. Epstein and A. MARden, 'Convex hulls in hyperbolic space, a theorem of Sullivan, and measured pleated surfaces', Analytical and geometric aspects of hyperbolic space (ed. D. B. A. Epstein), London Mathematical Society Lecture Note Series 111 (Cambridge University Press, 1987) 113-253.

2. D. B. A. Epstein, A. Marden and V. Markovic, 'Quasiconformal homeomorphisms and the convex hull boundary', Ann. of Math. (2) 159 (2004) 305-336.

3. D. B. A. EPSTEIn and V. MARKOVIC, 'The logarithmic spiral: a counter-example to the $k=2$ conjecture', Ann. of Math. (2) 161 (2005) 925-957.

4. R. Fehlman, 'Über extremale quasikonforme abbildungen', Comment. Math. Helv. 56 (1981) $558-580$.

5. N. LAKIC, 'Substantial boundary points for plane domains and Gardiner's conjecture', Ann. Acad. Sci. Fenn. 25 (2000) 285-306.

6. Ch. Pommerenke, Boundary behaviour of conformal maps (Springer, Berlin, 1992).

7. E. REICH, 'Extremal quasiconformal mappings of the disk', Geometric function theory, Vol. 1, Handbook of complex analysis (ed. R. Kühnau; Elsevier, Amsterdam, 2001) 75-136.

D. B. A. Epstein and V. Markovic

Mathematics Institute

University of Warwick

Coventry

CV4 7AL

United Kingdom

dbae@maths.warwick.ac.uk

markovic@maths.warwick.ac.uk
A. Marden

Mathematics Department

University of Minnesota

Minneapolis

MN 55455

USA

am@math.umn.edu 